\title{
DEFORMING A PL SUBMANIFOLD OF EUCLIDEAN SPACE INTO A HYPERPLANE
}

\author{
JOŽE VRABEC
}

\begin{abstract}
Let $M$ be a closed, $k$-connected, $m$-dimensional PL submanifold of $\mathbf{R}^{2 m-k-1}(1 \leq k \leq m-4)$. The main result of this paper states that if $m-k$ is even, then every embedding of $M$ into $\mathbf{R}^{2 m-k}$ can be isotopically deformed into $\mathbf{R}^{2 m-k-1}$, and specifies which embeddings of $M$ into $\mathbf{R}^{2 m-k}$ can be deformed into $\mathbf{R}^{2 m-k-1}$ in case $m-k$ is odd.
\end{abstract}

\section{INTRODUCTION}

For arbitrary polyhedra $X$ and $Y$ let $\mathscr{E}(X, Y)$ denote the set of PL embeddings of $X$ into $Y$ and let $\mathscr{J}(X, Y)$ be the set of PL isotopy classes of such embeddings.

Let $M$ be a closed PL submanifold of a Euclidean space $\mathbf{R}^{q}$. If we identify $\mathbf{R}^{q}$, as usual, with the hyperplane $\mathbf{R}^{q} \times 0$ in $\mathbf{R}^{q+1}$, every embedding of $M$ in $\mathbf{R}^{q}$ is at the same time an embedding in $\mathbf{R}^{q+1}$. Clearly, the inclusion $i: \mathscr{E}\left(M, \mathrm{R}^{q}\right) \hookrightarrow \mathscr{E}\left(M, \mathrm{R}^{q+1}\right)$ induces a function

$$
i_{*}: \mathscr{I}\left(M, \mathbf{R}^{q}\right) \rightarrow\left(M, \mathbf{R}^{q+1}\right),
$$

and this function is the object of our study. The most interesting problems concerning $i_{*}$ are to determine its range (i.e. to decide which embeddings of $M$ in $\mathbf{R}^{q+1}$ can be isotopically deformed into $\mathbf{R}^{q}$ ) and its point inverses (i.e. which pairs of embeddings into $\mathbf{R}^{q}$ are isotopic in $\mathbf{R}^{q+1}$ ).

Of course, this problem is too hard to hope for a concrete general solution. The oldest specific result of this kind that the author knows of is due to Tindell [13], who proved that if $M$ is a product of two spheres, then at least for some $q$ the function $i_{*}$ is not constant.

We shall take for $M$ a $k$-connected manifold and $q$ will be the smallest number such that the set $\mathscr{I}\left(M, \mathbf{R}^{q+1}\right)$ is (in general) nondegenerate, i.e. $q=$ $2 m-k-1$ [14]. The same problem in the smooth category (beside the analogous problem for immersions) was treated by Rigdon in his dissertation [9]. He

Received by the editors September 28, 1987.

1980 Mathematics Subject Classification (1985 Revision). Primary 57Q37, 57Q35.

Key words and phrases. PL manifold, embedding, immersion, isotopy, concordance, Euclidean space, embedding space, suspension of disk embeddings.

Research supported in part by the Research Council of Slovenia, contract numbers G-101/268176 and G-101/8229-78. 
determined the image of $i_{*}$ under the restriction $0 \leq 2 k \leq m-5$. Apparently his results have not been published. A proof of Rigdon's theorem for $k=0$, $m \geq 6$ is contained in Yasui's paper [16].

Our restriction is $1 \leq k \leq m-4$, and our main result (Corollary 3.2) is a PL version of Rigdon's theorem on the image of $i_{*}$. In particular, $i_{*}$ is surjective if and only if either $m-k$ is even or $2 \cdot H_{k+1}(M)=0$, and $i_{*}$ is constant if and only if either $m-k$ is even and $H_{k+1}\left(M ; \mathbb{Z}_{2}\right)=0$ (in which case $M$ unknots in $\mathrm{R}^{2 m-k}$, by [14]) or $m-k$ is odd and $H_{k+1}(M)$ has no 2-torsion. We also prove (Corollary 3.3) that two embeddings of $M$ in $\mathbb{R}^{2 m-k-1}$ are isotopic in $\mathbf{R}^{2 m-k}$ if and only if their restrictions to $M-\left(m\right.$-disk) are isotopic in $\mathbf{R}^{2 m-k-1}$.

We shall conclude this introduction by fixing our notation and terminology. We work throughout in the PL category and all our terms (manifold, map, homeomorphism, etc.) must be interpreted accordingly. We refer to Cohen [2] for regular neighborhood theory and to Hudson [4] for the rest of standard PL topology.

Let $M$ and $Q$ be manifolds. An embedding $f: M \rightarrow Q$ is proper if it is a proper map and $f^{-1}(\partial Q)=\partial M$; if $M \subset Q$ and the inclusion is a proper embedding, we say that $M$ is a proper submanifold of $Q$.

If $f_{0}, f_{1}: M \rightarrow Q$ are embeddings, then a concordance of $M$ in $Q$ from $f_{0}$ to $f_{1}$ is an embedding $F: I \times M \rightarrow I \times Q$ (where $I:=[0,1]$ ) such that $F^{-1}(i \times Q)=i \times M$ and $F(i, x)=\left(i, f_{i}(x)\right)$ for $i=0,1$ and $x \in M$. The concordance $F$ is fixed on $A \subset M$ (or keeps $A$ fixed) if $F(t, a)=\left(t, f_{0}(a)\right.$ ) for each $t \in I$ and $a \in A$; in this case we also say that $F$ is a concordance $\operatorname{rel} A$.

An isotopy of $M$ in $Q$ is a concordance of $M$ in $Q$ that is level preserving, i.e. $F(t \times M) \subset t \times Q$ for each $t \in I$; we shall consistently denote by $F_{t}$ the embedding $M \rightarrow Q$ such that $F(t, x)=\left(t, F_{t}(x)\right) \quad(t \in I, x \in M)$. A concordance or isotopy is proper if it is a proper embedding.

An isotopy of $Q$ is a proper isotopy $F$ of $Q$ in $Q$ such that $F_{0}=$ id. The isotopy $F$ carries $A \subset Q$ onto $B \subset Q$ if $F_{1}(A)=B$. $F$ moves the embedding $f: M \rightarrow Q$ to the embedding $g: M \rightarrow Q$ if $g=F_{1} f$.

For each positive integer $n$ we shall denote by $\mathbf{R}^{n}$ the $n$-dimensional Euclidean space (and $\left.\mathbf{R}^{0}:=\{0\}\right) ; 0^{n}$ will be the origin of $\mathbb{R}^{n}, D^{n}$ the product of $n$ copies of the interval $[-1,1]$ (we never write $D$ for $D^{1}$ ), and $S^{n-1}$ the boundary of $D^{n}$. For each integer $k>0$ we identify $\mathbf{R}^{n}$ with the subspace $\mathbf{R}^{n} \times 0^{k}$ of $\mathbf{R}^{n+k}$, and correspondingly we think of $D^{n}$ and $S^{n-1}$ as lying in $D^{n+k}$ and $S^{n+k-1}$, respectively.

Let $\mathbf{R}_{+}:=[0, \infty)$ and $\mathbf{R}_{-}:=(-\infty, 0]$, and for $i=+,-$ let $\mathbf{R}_{i}^{n}:=\mathbf{R}^{n-1} \times \mathbf{R}_{i}$ and $S_{i}^{n}:=\mathbf{R}_{i}^{n+1} \cap S^{n}$. In $\S 1$ we shall also use the two hemispheres in the direction of the first coordinate: let $S_{1, i}^{n}:=\left(\mathbf{R}_{i} \times \mathbf{R}^{n}\right) \cap S^{n}(i=+,-)$.

Let $Q$ be an oriented $q$-manifold. If $a$ and $b$ are integral PL chains (e.g. orientcd compact manifolds) in $Q$ such that their dimensions add up to $q$ and 
their supports $|a|$ and $|b|$ intersect transversely, define $\operatorname{sc}(a, b ; Q)$, the intersection number of $a$ with $b$ in $Q$, as usual by adding up the local intersection indices of $a$ with $b$ at all points of $|a| \cap|b|$. If $a$ and $b$ are not transverse but satisfy the weaker conditions

$$
\begin{gathered}
|\partial a| \cap|\partial b|=\varnothing, \\
|a| \cap \mathrm{Cl}(|\partial b| \cap \operatorname{int} Q)=\varnothing, \\
|b| \cap \mathrm{Cl}(|\partial a| \cap \operatorname{int} Q)=\varnothing,
\end{gathered}
$$

define $\operatorname{sc}(a, b ; Q)$ as follows: choose mutually transverse chains $a^{\prime}, b^{\prime}$ such that $a^{\prime}$ approximates $a$ (in the sense that $\partial a^{\prime}=\partial a$ and the cycle $a^{\prime}-a$ bounds in $Q)$ and $b^{\prime}$ approximates $b$ and then $\operatorname{set} \operatorname{sc}(a, b ; Q):=\operatorname{sc}\left(a^{\prime}, b^{\prime} ; Q\right)$.

Whenever the intersection number function sc involves one of the manifolds $\mathbf{R}^{n}, D^{n}$, or $S^{n}$, we assume these manifolds oriented in some standard way; the actual choice of "standard" is not of much consequence since we shall not be much interested in the signs.

A codimension 0 submanifold of an oriented manifold will normally be assumed to have the induced orientation.

When we write homology (or chain) groups without specifying the coefficients, integer coefficients are understood.

\section{HOMOTOPY GROUPS OF PL BLOCK EMBEDDING SPACES OF SPHERES INTO SPHERES}

Some of the groups of the title will be coefficient groups in our main theorems. In this section we shall therefore explain the necessary facts. We shall give a definition of homotopy groups of PL block embedding spaces without mentioning the embedding spaces themselves.

Let $Q$ be a compact $q$-manifold and $M$ a compact proper $m$-submanifold of $Q$. For an arbitrary positive integer $p$ denote by $\Omega_{p} E(M, Q)$ the set of all locally flat proper embeddings $e: D^{p} \times(M, \partial M) \rightarrow D^{p} \times(Q, \partial Q)$ (i.e. $e$ is a proper embedding on each member of the pair) such that $e \mid S^{p-1} \times M=$ id ; let $\pi_{p} E(M, Q)$ be the set of locally flat proper concordance classes $\left(\operatorname{rel} S^{p-1} \times M\right)$ in $\Omega_{p} E(M, Q)$ (here we have in mind concordance of pairs, of course). We shall be interested only in the cases with $q-m \geq 3$; then every embedding of $D^{p} \times(M, \partial M)$ in $D^{p} \times(Q, \partial Q)$ is locally flat, all concordances between such embeddings are locally flat, and concordance classes are the same as isotopy classes or ambient isotopy classes. For an arbitrary $e$ in $\Omega_{p} E(M, Q)$ we shall denote by $[e] \in \pi_{p} E(M, Q)$ the class of $e$. In what follows we shall often write $\Omega_{p}$ for $\Omega_{p} E(M, Q)$ and $\pi_{p}$ for $\pi_{p} E(M, Q)$.

Let $f: D^{p} \rightarrow D^{p}$ be any embedding. For an arbitrary $e \in \Omega_{p}$ denote by $e / f$ the mapping $D^{p} \times M \rightarrow D^{p} \times Q$ which agrees with $\left(f \times \mathrm{id}_{Q}\right) e\left(f \times \mathrm{id}_{M}\right)^{-1}$ on $f\left(D^{p}\right) \times M$ and is the identity elsewhere. Clearly $e / f \in \Omega_{p}$. 
1.1. Lemma. If $f: D^{p} \rightarrow D^{p}$ is an orientation preserving embedding, then $[e / f]=[e]$ for each $e \in \Omega_{p}$.

This follows easily from the fact that $f$ is isotopic to the identity.

Define $g_{+}, g_{-}: D^{p} \rightarrow D^{p}$ by

$$
g_{ \pm}\left(t_{1}, \ldots, t_{p}\right):=\left(\frac{1}{2}\left(t_{1} \pm 1\right), t_{2}, \ldots, t_{p}\right) .
$$

For any $e_{1}, e_{2} \in \Omega_{p}$ let $e_{1}+e_{2}: D^{p} \times M \rightarrow D^{p} \times Q$ be the embedding which agrees with $e_{1} / g_{-}$on $[-1,0] \times D^{p-1} \times M$ and with $e_{2} / g_{+}$on $[0,1] \times D^{p-1} \times$ $M$. Obviously, $e_{1}+e_{2} \in \Omega_{p}$, and $\left[e_{1}+e_{2}\right]$ depends only on $\left[e_{1}\right]$ and $\left[e_{2}\right]$. This means that + is a binary operation on $\Omega_{p}$ and that it induces a binary operation, also denoted + , in $\pi_{p}$. It can easily be deduced from Lemma 1.1 that the latter operation makes $\pi_{p}$ a group and that for $p>1$ this group is abelian (the zero element is the class of the inclusion $D^{p} \times M \hookrightarrow D^{p} \times Q$, and for any $e \in \Omega_{p}$ and any orientation reversing embedding $f: D^{p} \rightarrow D^{p},[e / f]$ is the opposite of $[e])$. In fact, this is the $p$ th homotopy group of the $\Delta$-set of "block embeddings" of $(M, \partial M)$ into $(Q, \partial Q)$ (cf. Morlet [8, p. 343] and Burghelea, Lashof, and Rothenberg [1, pp. 121-122]).

In applications $(\S \S 2-3)$ we shall be interested only in the case $(Q, M)=$ $\left(D^{q}, D^{m}\right)$ with $q-m \geq 3$. In calculations we may replace the pair $\left(D^{q}, D^{m}\right)$ by $\left(S^{q-1}, S^{m-1}\right)$. Indeed, using the familiar conewise extension of embeddings and isotopies we can prove:

1.2. Lemma. Let $1 \leq m \leq q-3$. The function which assigns to each $e \cdot \in$ $\Omega_{p} E\left(D^{m}, D^{q}\right)$ its restriction to $D^{p} \times S^{m-1}$ induces an isomorphism

$$
\rho: \pi_{p} E\left(D^{m}, D^{q}\right) \rightarrow \pi_{p} E\left(S^{m-1}, S^{q-1}\right) .
$$

The conewise extensions to which we alluded above are identity on $D^{p} \times 0^{m}$ or $I \times D^{p} \times 0^{m}$, respectively. Therefore the proof of 1.2 gives at the same time the following:

1.3. Corollary. Each element of $\pi_{p} E\left(D^{m}, D^{q}\right)$ can be represented by an embedding $D^{p+m} \rightarrow D^{p+q}$ in $\Omega_{p} E\left(D^{m}, D^{q}\right)$ which is identity on $D^{p} \times 0^{m}$. If $e_{1}, e_{2} \in \Omega_{p} E\left(D^{m}, D^{q}\right)$ are both identity on $D^{p} \times 0^{m}$ and if $\left[e_{1}\right]=\left[e_{2}\right]$, then there exists a proper isotopy from $e_{1}$ to $e_{2}$ which is fixed on $D^{p} \times 0^{m}$ (and $\left.S^{p-1} \times D^{m}\right)$.

This means that $\pi_{p} E\left(D^{m}, D^{q}\right)=\pi_{p}\left(\tilde{V}_{q, m}\right)$, where $\tilde{V}_{q, m}$ is the block PL Stiefel manifold of Rourke and Sanderson [10, p. 274].

Take any $e: D^{p} \times D^{m} \rightarrow D^{p} \times D^{q}$ belonging to $\Omega_{p} E\left(D^{m}, D^{q}\right)$; let e.g. $e(x, y)=(f(x, y), g(x, y))$, where $f$ and $g$ map $D^{p} \times D^{m}$ into $D^{p}$ and $D^{q}$, respectively. The map $e^{\#}: D^{p} \times D^{1} \times D^{m} \rightarrow D^{p} \times D^{1} \times D^{q}$ defined by $e^{\#}(x, t, y):=(f(x, y), t, g(x, y))$ obviously belongs to $\Omega_{p} E\left(D^{m+1}, D^{q+1}\right)$, 
and $\left[e^{\#}\right]$ depends only on $[e]$. Moreover, the function

$$
\Sigma: \pi_{p} E\left(D^{m}, D^{q}\right) \rightarrow \pi_{p} E\left(D^{m+1}, D^{q+1}\right)
$$

defined by $\Sigma[e]:=\left[e^{\#}\right]$ is a homomorphism; we shall call it the suspension homomorphism because it is induced by the suspension map of embedding spaces as defined by Husch [6] in greater generality. Define

$$
\sigma: \pi_{p} E\left(S^{m-1}, S^{q-1}\right) \rightarrow \pi_{p} E\left(S^{m}, S^{q}\right),
$$

also called the suspension homomorphism, by $\sigma:=\rho \Sigma \rho^{-1}$. For any $n$ let $\psi: \mathbf{R}^{n} \rightarrow \mathbf{R}^{n+1}$ be the shift map: $\psi(x):=(0, x)$. Unknotting of codimension 3 disk pairs implies:

1.4. Lemma. Suppose that $1 \leq m \leq q-3$. Let $e \in \Omega_{p} E\left(S^{m-1}, S^{q-1}\right)$. If $e^{\prime}: D^{p} \times S^{m} \rightarrow D^{p} \times S^{q}$ from $\Omega_{p} E\left(S^{m}, S^{q}\right)$ agrees with $(\mathrm{id} \times \psi) e(\mathrm{id} \times \psi)^{-1}$ on $D^{p} \times\left(0 \times S^{m-1}\right)$ and maps the hemispheres $D^{p} \times S_{1,+}^{m}$ and $D^{p} \times S_{1,-}^{m}$ into $D^{p} \times S_{1,+}^{q}$ and $D^{p} \times S_{1,-}^{q}$, respectively, then $\left[e^{\prime}\right]=\sigma[e]$.

Husch [6] proved:

1.5. Proposition. If $1 \leq m \leq q-3$, then the suspension homomorphisms $\Sigma: \pi_{p} E\left(D^{m}, D^{q}\right) \rightarrow \pi_{p} E\left(D^{m+1}, D^{q+1}\right)$ and

$$
\sigma: \pi_{p} E\left(S^{m-1}, S^{q-1}\right) \rightarrow \pi_{p} E\left(S^{m}, S^{q}\right)
$$

are surjective for $q \geq p+1$ and bijective for $q>p+1$.

Proof. Husch obtained this (in the more general situation) by nontrivial arguments from the exact homotopy sequences of certain Kan fibrations. In our situation, however, the proof is very simple. Let $q \geq p+1$ and take any $e: D^{p} \times S^{m} \rightarrow D^{p} \times S^{q}$ from $\Omega_{p} E\left(S^{m}, S^{q}\right)$. Since $D^{p} \times S^{q}$ is $p$-connected and since $D^{p}$ unknots ( $\mathrm{rel} S^{p-1}$ ) in $D^{p} \times S^{q}$, by Zeeman's Unknotting Theorem, there is an $e^{\prime}$ in [e] such that $e^{\prime} \mid D^{p} \times 1 \times 0^{m}=\mathrm{id}$. Because of uniqueness of relative regular neighborhoods we can now deform $e^{\prime}$ to an $e^{\prime \prime} \in[e]$ which embeds $D^{p} \times S_{1, i}^{m}$ properly into $D^{p} \times S_{1, i}^{q}(i=+,-)$. By Lemma 1.4 this implies that $[e]=\left[e^{\prime \prime}\right]$ is in the image of $\sigma$.

Injectivity of $\sigma$ when $q>p+1$ is proved by essentially the same argument; we only replace $D^{p}$ by $I \times D^{p}$.

Proposition 1.5 is very useful at studying the groups $\pi_{p} E\left(S^{m}, S^{q}\right)$ with $p$ approximately equal to $q-m$, for it reduces the problem to a few cases with very small $m$, which are usually easier to handle than the general case. For example, we have the following proposition (cf. $[11,2.8]$ ), which is a direct consequence of the two subsequent lemmas.

1.6. Proposition. If $q \geq 3$ and $2 q \geq p+3$, then $\pi_{p} E\left(S^{0}, S^{q}\right) \approx \pi_{p}\left(S^{q}\right)$.

In the following two lemmas, the number $1 \in \mathbf{R}$ is identified with $1 \times 0^{n} \in$ $\mathbf{R}^{n+1}$ for any $n$ (similarly for the number -1 ). 
1.7. Lemma. If $q \geq 3$ and $2 q \geq p+3$, then the function which assigns to each embedding $e: D^{p} \times 1 \rightarrow D^{p} \times S^{q}$ in $\Omega_{p} E\left(1, S^{q}\right)$ the composite

$$
\left(D^{p}, S^{p-1}\right) \stackrel{\equiv}{\rightarrow}\left(D^{p} \times 1, S^{p-1} \times 1\right) \stackrel{e}{\rightarrow}\left(D^{p} \times S^{q}, S^{p-1} \times 1\right) \stackrel{\text { proj }_{2}}{\rightarrow}\left(S^{q}, 1\right),
$$

induces an isomorphism $\pi_{p} E\left(1, S^{q}\right) \rightarrow \pi_{p}\left(S^{q}, 1\right)$.

Proof. The map in question is easily seen to be a homomorphism. The inverse homomorphism is induced by the function which assigns to each map $f:\left(D^{p}, S^{p-1}\right) \rightarrow\left(S^{q}, 1\right)$ the embedding $D^{p} \times 1 \rightarrow D^{p} \times S^{q}$ given by $(x, 1) \mapsto$ $(x, f(x))$. (The proposed inverse is really a right inverse for all $p$ and $q$, and is a left inverse if $D^{p}$ unknots in $D^{p} \times S^{q}$, i.e. if $q \geq 3$ and $2 q \geq p+3$.)

1.8. Lemma. If $q \geq 3$, then the restriction induced homomorphism $\pi_{p} E\left(S^{0}, S^{q}\right)$ $\rightarrow \pi_{p} E\left(1, S^{q}\right)$ is an isomorphism.

Proof. Denote this restriction homomorphism by $r$. To prove injectivity of $r$ take any embedding $e \in \Omega_{p} E\left(S^{0}, S^{q}\right)$ such that $e \mid D^{p} \times 1$ is isotopic (rel $\partial$ ) to the inclusion. By the isotopy extension theorem there exists an isotopy $F$ of $D^{p} \times S^{q}$, fixed on the boundary, such that $F_{1} e \mid D^{p} \times 1=\mathrm{id}$. Then $F_{1} e \mid D^{p} \times(-1)$ is an embedding, on the boundary equal to the inclusion, of $D^{p} \times(-1)$ into $D^{p} \times S^{q}-D^{p} \times 1 \approx D^{p} \times \mathbb{R}^{q}$. Therefore $F_{1} e \mid D^{p} \times(-1)$, and hence $e$, are isotopic ( rel $\partial$ ) to the inclusion.

To prove surjectivity of $r$ take any $e \in \Omega_{p} E\left(1, S^{q}\right)$; we have to show that $S^{p-1} \times(-1)$ bounds a $p$-disk in $M:=\left(D^{p} \times S^{q}\right)-e\left(D^{p} \times 1\right)$. But this follows from Irwin's Embedding Theorem since $M$ is contractible: one can show by a general position argument that $M$ is 1 -connected and by standard methods of homology theory that $\tilde{H}_{*}(M)=0$.

Propositions 1.6 and 1.5 and Lemma 1.2 imply the following well-known theorem (cf. [11, 2.11]).

1.9. Theorem. If $q-m \geq \max \{p+1,3\}$, then

$$
\pi_{p} E\left(S^{m-1}, S^{q-1}\right)=\pi_{p} E\left(D^{m}, D^{q}\right)=0 .
$$

The main objective of this section is to calculate the groups $\pi_{p} E\left(S^{m-1}, S^{q-1}\right)$ $=\pi_{p} E\left(D^{m}, D^{q}\right)$ with $q-m=p \geq 3$. These are already nontrivial. In fact, they are equal to their orthogonal analogues $\pi_{p}\left(V_{q, m}\right)$, the homotopy groups of Stiefel manifolds, which are well known. For most cases this follows from $[11,2.11]$ or from results of Lashof [7]. However, in the sequel we shall need the explicit isomorphisms described in Theorems 1.13 and 1.15 below, and therefore we include direct, elementary proofs.

Let $p \geq 1$ and $m \geq 0$. Pick any $a \in S^{m+p}-S^{m}$. For each $e \in$ $\Omega_{p} E\left(S^{m}, S^{m+p}\right)$ set

$$
s(e):=\operatorname{sc}\left(D^{p} \times a, e\left(D^{p} \times S^{m}\right) ; D^{p} \times S^{m+p}\right) .
$$


Clearly $s(e)$ depends only on $[e]$, and therefore $s$ induces a function, also denoted by $s$, from $\pi_{p} E\left(S^{m}, S^{m+p}\right)$ to $Z$. It follows from the definition that the latter function is a homomorphism, independent of the point $a$. The following lemma, too, is a simple consequence of the definitions (cf. [14, pp. 149-150]).

1.11. Lemma. For each $m$ and $p$, the following diagram is commutative up to sign:

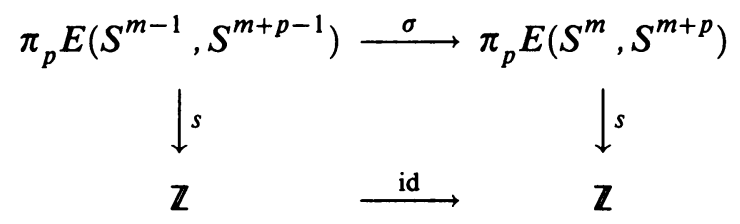

For $m=0$ we can similarly define a homomorphism $s_{+}: \pi_{p} E\left(S^{0}, S^{p}\right) \rightarrow \mathbf{Z}$ by assigning to each $e \in \Omega_{p} E\left(S^{0}, S^{p}\right)$ the number

$$
s_{+}(e):=\operatorname{sc}\left(D^{p} \times a, e\left(D^{p} \times 1\right) ; D^{p} \times S^{p}\right) .
$$

We can prove (see $[14$, p. 149]) that

$$
s=\left(1+(-1)^{p}\right) s_{+} \text {for } m=0 .
$$

We shall now describe the groups $\pi_{p} E\left(S^{m}, S^{m+p}\right)$. For any $e \in \Omega_{p} E\left(S^{0}, S^{p}\right)$ $s_{+}(e)$ is the degree of the map $\operatorname{proj}_{2} \circ e:\left(D^{p} \times 1, S^{p-1} \times 1\right) \rightarrow\left(S^{p}, 1\right)$. Therefore Lemmas 1.7 and 1.8 imply:

1.13. Theorem. If $p \geq 3$, then $s_{+}: \pi_{p} E\left(S^{0}, S^{p}\right) \rightarrow \mathbb{Z}$ is an isomorphism.

1.14. Corollary $[14,6.1]$. The homomorphism $s$ is trivial if $p$ is odd, and the image of $s$ is $2 Z$ if $p$ is even.

This follows from 1.13, (1.12), 1.5, and 1.11.

For the groups $\pi_{p} E\left(S^{m}, S^{m+p}\right)$ with $m>0$ we have

1.15. Theorem. Let $m>0$ and $p \geq 3$. If $p$ is even, then $s: \pi_{p} E\left(S^{m}, S^{m+p}\right) \rightarrow$ $2 Z$ is an isomorphism. If $p$ is odd, then $\pi_{p} E\left(S^{m}, S^{m+p}\right) \approx \mathbf{Z}_{2}$.

Proof. For even $p$ the theorem follows from 1.13, (1.12), 1.5, and 1.11 by induction on $m$.

Suppose that $p$ is odd. By 1.5 and 1.13 we only have to prove that the kernel of $\sigma: \pi_{p} E\left(S^{0}, S^{p}\right) \rightarrow \pi_{p} E\left(S^{1}, S^{p+1}\right)$ is mapped by $S_{+}$onto $2 Z$.

Take any $e: D^{p} \times S^{0} \rightarrow D^{p} \times S^{p}$ in $\Omega_{p} E\left(S^{0}, S^{p}\right)$. If we represent $\rho^{-1}[e]$ by the embedding $D^{p} \times D^{1} \rightarrow D^{p} \times D^{p+1}$ which is the conewise extension of $e$ (and of the inclusion of $S^{p-1} \times D^{1}$ into $S^{p-1} \times D^{p-1}$ ), we obtain a representative $e^{\prime}: D^{p} \times S^{1} \rightarrow D^{p} \times S^{p+1}$ of $\sigma[e]=\rho \Sigma \rho^{-1}[e]$ such that

$e^{\prime} \mid D^{p} \times\left(0 \times S^{0}\right)=(\mathrm{id} \times \psi) e(\mathrm{id} \times \psi)^{-1} \quad($ see Lemma 1.4$)$,

$e^{\prime}\left(\operatorname{int}\left(D^{p} \times S_{1, i}^{1}\right)\right) \subset \operatorname{int}\left(D^{p} \times S_{1, i}^{p+1}\right)$ for $i=+,-$, and 
$e^{\prime} \mid D^{p} \times\{u,-u\}=$ id, where $u:=1 \equiv(1,0) \equiv\left(1,0^{p+1}\right)$.

Now suppose that $\sigma[e]=0$. Then there exists an isotopy $F$ of $D^{p} \times S^{p+1}$, fixed on the boundary, such that for some $\delta>0$ we have $F_{t}=$ id if $0 \leq t \leq \delta$ and $F_{t} \mid D^{p} \times S^{1}=e^{\prime}$ if $1-\delta \leq t \leq 1$.

Beside $u$ consider the points $v:=(0,1) \equiv\left(0,1,0^{p}\right)$ and $a:=\left(0^{p+1}, 1\right)$ in $S^{p+1}$. Let $L:=\left\{\left(x, 0^{p}, y\right) \in S^{p+1} \mid y \geq 0\right\}$; thus $L$ is the "upper" half of the 1 -sphere $S^{1}$ in the coordinate plane spanned by $u$ and $a$. Note that $a$ is the midpoint of $L$ and $\partial L=\{u,-u\}=S^{0}$. Using the properties of $e^{\prime}$ and $F$ stated above we can calculate $s_{+}(e)$ as follows:

$$
\begin{aligned}
s_{+}(e) & =\operatorname{sc}\left(D^{p} \times\left(0^{p}, 1\right), e\left(D^{p} \times u\right) ; D^{p} \times S^{p}\right) \\
& =\operatorname{sc}\left(D^{p} \times a, e^{\prime}\left(D^{p} \times v\right) ; D^{p} \times 0 \times S^{p}\right) \\
& = \pm \operatorname{sc}\left(D^{p} \times L, e^{\prime}\left(D^{p} \times v\right) ; D^{p} \times S^{p+1}\right) \\
& = \pm \operatorname{sc}\left(I \times D^{p} \times L, F\left(\delta \times D^{p} \times v\right) ; I \times D^{p} \times S^{p+1}\right) \\
& = \pm \operatorname{sc}\left(I \times D^{p} \times L, \partial\left(F\left([\delta, 1-\delta] \times D^{p} \times v\right)\right) ; I \times D^{p} \times S^{p+1}\right) \\
& = \pm \operatorname{sc}\left(\partial\left(I \times D^{p} \times L\right), F\left([\delta, 1-\delta] \times D^{p} \times v\right) ; I \times D^{p} \times S^{p+1}\right) \\
& = \pm \operatorname{sc}\left(I \times D^{p} \times \partial L, F\left([\delta, 1-\delta] \times D^{p} \times v\right) ; I \times D^{p} \times S^{p+1}\right) \\
& = \pm \operatorname{sc}\left(F^{-1}\left(I \times D^{p} \times \partial L\right),[\delta, 1-\delta] \times D^{p} \times v ; I \times D^{p} \times S^{p+1}\right) \\
& = \pm \operatorname{sc}\left(F^{-1}\left(I \times D^{p} \times S^{0}\right), I \times D^{p} \times v ; I \times D^{p} \times S^{p+1}\right) \\
& = \pm \operatorname{sc}\left(I \times D^{p} \times v, F^{-1}\left(I \times D^{p} \times S^{0}\right) ; I \times D^{p} \times S^{p+1}\right) .
\end{aligned}
$$

Now,

$$
F^{-1}: I \times D^{p} \times S^{0} \rightarrow I \times D^{p} \times S^{p+1}
$$

is essentially an element of $\Omega_{p+1} E\left(S^{0}, S^{p+1}\right)$. Since $p+1$ is even, Corollary 1.14 implies that the number in the last line of the above calculation is even. We have thus proved that $s_{+}(\operatorname{ker} \sigma) \subset 2 \mathbb{Z}$.

Conversely, it follows from 1.14 that for each integer $n$ there exists an embedding $g: I \times D^{p} \times S^{0} \rightarrow I \times D^{p} \times S^{p+1}$ such that $g \mid \partial\left(I \times D^{p}\right) \times S^{0}=$ id and

$$
\operatorname{sc}\left(I \times D^{p} \times v, g\left(I \times D^{p} \times S^{0}\right) ; I \times D^{p} \times S^{p+1}\right)=2 n .
$$

We can even choose $g$ level preserving with respect to the first factor $I$, and then we can extend $g$ to an isotopy $G$ of $D^{p} \times S^{p+1}$, fixed on the boundary. Since $G_{1}$ is identity on $S^{p-1} \times S^{p+1}$ and $D^{p} \times S^{0}$ there exists, by uniqueness of regular neighborhoods, an isotopy $G^{\prime}$ of $D^{p} \times S^{p+1}$ which keeps the boundary and $D^{p} \times S^{0}$ fixed and carries $G_{1}^{-1}\left(D^{p} \times S_{1, i}^{p+1}\right)$ onto $D^{p} \times S_{1, i}^{p+1} \quad(i=+,-)$. If we set $G^{\prime} G^{-1}=: F$, we are in the previous situation: $e^{\prime}:=F_{1} \mid D^{p} \times S^{1}$ is in $\Omega_{p} E\left(S^{1}, S^{p+1}\right), e:=(\operatorname{id} \times \psi)^{-1} e^{\prime}(\operatorname{id} \times \psi)$ is in $\Omega_{p} E\left(S^{0}, S^{p}\right)$, and by construction we have $\sigma[e]=\left[e^{\prime}\right]=0$ (see Lemma 1.4). Moreover, as shown 
above,

$$
\begin{aligned}
s_{+}(e) & = \pm \operatorname{sc}\left(I \times D^{p} \times v, F^{-1}\left(I \times D^{p} \times S^{0}\right) ; I \times D^{p} \times S^{p+1}\right) \\
& = \pm \operatorname{sc}\left(I \times D^{p} \times v, g\left(I \times D^{p} \times S^{0}\right) ; I \times D^{p} \times S^{p+1}\right) \\
& = \pm 2 n .
\end{aligned}
$$

This proves that $2 \mathbb{Z} \subset s_{+}(\operatorname{ker} \sigma)$ and concludes the proof of Theorem 1.15.

1.16. Lemma. If $p \geq 3$, then for any positive integer $m$ each element of $\pi_{p} E\left(D^{m}, D^{m+p}\right)$ can be represented by a level preserving embedding, i.e. an embedding $D^{p} \times D^{m} \rightarrow D^{p} \times D^{m+p}$ of the form $(x, y) \mapsto(x, \varphi(x, y)$ ) (for $a$ suitable $\left.\varphi: D^{p} \times D^{m} \rightarrow D^{m+p}\right)$.

The reader can find much deeper results of this sort e.g. in Lashof [7]. Here we give a short proof of the stated simple version, which will be sufficient for our purposes.

Proof. As shown in the proof of Lemma 1.7, an arbitrary element of $\pi_{p} E\left(1, S^{p}\right)$ has a level preserving representative $f: D^{p} \times 1 \rightarrow D^{p} \times S^{p}$, say $f(x, 1)=$ $(x, \varphi(x))$. The latter extends to a level preserving embedding $D^{p} \times D^{1} \rightarrow$ $D^{p} \times D^{p+1}$ in $\Omega_{p} E\left(D^{1}, D^{p+1}\right)$ defined by $(x, y) \mapsto(x, y \cdot \varphi(x))$. Hence each element of $\pi_{p} E\left(D^{1}, D^{p+1}\right)$ has a level preserving representative, by Lemmas 1.8 and 1.2. Since, by 1.5 , a repeated suspension homomorphism maps $\pi_{p} E\left(D^{1}, D^{p+1}\right)$ onto $\pi_{p} E\left(D^{m}, D^{m+p}\right)$, each element of the latter group has a level preserving representative.

We shall now give a new description of an isomorphism of $\pi_{p} E\left(S^{m}, S^{m+p}\right)$ onto $\mathbb{Z}$ or $\mathbb{Z}_{2}$. An arbitrary embedding $e \in \Omega_{p} E\left(S^{m}, S^{m+p}\right)$ can also be interpreted as an element of $\Omega_{p} E\left(S^{m}, S^{m+p+1}\right)$. By Theorem 1.9, $e$ represents the zero element of $\pi_{p} E\left(S^{m}, S^{m+p+1}\right)$, and hence there exists a proper isotopy $F$ of $D^{p} \times S^{m}$ in $D^{p} \times S^{m+p+1}$ (rel $\left.\partial\right)$ from $e$ to the inclusion. For a given $\boldsymbol{e}$ there are many such isotopies (even essentially different ones), but they all have the same intersection number (in $\mathbb{Z}$ or $\left.\mathbb{Z}_{2}\right)$ with a "straight" $(p+1)$-disk. More precisely:

1.17. Proposition. Let $p \geq 3, m \geq 0, d:=1-(-1)^{p}$, and $\mathbb{Z}_{d}:=\mathbb{Z} / d \mathbb{Z} \quad(=\mathbb{Z}$ or $\left.\mathbb{Z}_{2}\right)$. There exists a unique surjective homomorphism $\lambda: \pi_{p} E\left(S^{m}, S^{m+p}\right) \rightarrow$ $\mathbb{Z}_{d}$ such that for any $e \in \Omega_{p}\left(S^{m}, S^{m+p}\right)$ and any proper concordance $F$ of $D^{p} \times S^{m}$ in $D^{p} \times S^{m+p+1}($ rel $\partial)$ with $F_{0}=e$ and $F_{1}=$ id the following relation holds, where $a:=\left(0^{m+p+1}, 1\right) \in S^{m+p+1}$ :

$$
\lambda[e]=\operatorname{sc}\left(I \times D^{p} \times a, F\left(I \times D^{p} \times S^{m}\right) ; I \times D^{p} \times S^{m+p+1}\right) \bmod d .
$$

If $m>0$ or if $p$ is even, $\lambda$ is an isomorphism.

Proof. Note that the second assertion of this proposition follows from the first one since $\pi_{p} E\left(S^{m}, S^{m+p}\right) \approx \mathbf{Z}_{d}$ unless $m=0$ and $p$ is odd. 
We shall write $m+p+1=: q$. For an arbitrary proper concordance $F: I \times$ $\left(D^{p} \times S^{m}\right) \rightarrow I \times\left(D^{p} \times S^{q}\right)$ which satisfies the conditions

$$
F \mid I \times S^{p-1} \times S^{m}=\mathrm{id}, \quad F_{0}\left(D^{p} \times S^{m}\right) \subset D^{p} \times S^{q-1}, \quad F_{1}=\mathrm{id},
$$

let

$$
\mu(F):=\operatorname{sc}\left(I \times D^{p} \times a, F\left(I \times D^{p} \times S^{m}\right) ; I \times D^{p} \times S^{q}\right) .
$$

Let $G$ be another such concordance, suppose that $G_{0}=F_{0}$, and compare $\mu(G)$ with $\mu(F)$. Define

$$
H: D^{1} \times D^{p} \times S^{m} \rightarrow D^{1} \times D^{p} \times S^{q}
$$

by

$$
H(t, x, y):= \begin{cases}F(t, x, y), & \text { if } t \geq 0, \\ r_{1} G r_{1}(t, x, y), & \text { if } t \leq 0,\end{cases}
$$

where $r_{1}:=(-\mathrm{id}) \times \mathrm{id} \times \mathrm{id}: D^{1} \times D^{p} \times S^{q} \rightarrow D^{1} \times D^{p} \times S^{q}$. Then $H \in$ $\Omega_{p+1} E\left(S^{m}, S^{q}\right)$, and therefore Corollary 1.14 implies that

$$
s(H)=\operatorname{sc}\left(D^{p+1} \times a, H\left(D^{p+1} \times S^{m}\right) ; D^{p+1} \times S^{q}\right) \in d \mathbb{Z} .
$$

On the other hand it is easy to see that $s(H)=\mu(F)-\mu(G)$. This means that the residue class mod $d$ of $\mu(F)$ depends only on $F_{0}$, i.e. the function $\mu$ induces a function $\lambda^{\prime}: \Omega_{p} E\left(S^{m}, S^{q-1}\right) \rightarrow \mathbb{Z}_{d}$.

Take embeddings $e^{\prime}, e \in \Omega_{p} E\left(S^{m}, S^{q-1}\right)$ representing the same element of $\pi_{p} E\left(S^{m}, S^{q-1}\right)$. If we choose a proper isotopy $G$ of $D^{p} \times S^{m}$ in $D^{p} \times S^{q-1}$ with $G_{0}=e^{\prime}$ and $G_{1}=e$, and a proper isotopy $F$ of $D^{p} \times S^{m}$ in $D^{p} \times S^{q}$ with $F_{0}=e$ and $F_{1}=$ id (both isotopies keeping the boundary fixed), then the concatenation $F^{\prime}$ of $G$ and $F$ satisfies $\mu\left(F^{\prime}\right)=\mu(F)$ (because the image of $G$ misses $\left.I \times D^{p} \times a\right)$, and consequently $\lambda^{\prime}\left(e^{\prime}\right)=\lambda^{\prime}(e)$. Therefore $\lambda^{\prime}$ induces a function $\lambda: \pi_{p} E\left(S^{m}, S^{q-1}\right) \rightarrow \mathbb{Z}_{d}$. A similar simple argument shows that $\lambda$ is a homomorphism.

Surjectivity of $\lambda$ will be proved by induction on $m$. Take first $m=0$. Let $u:=\left(1,0^{p}\right) \in S^{p}$ and take a degree 1 map $f:\left(D^{p}, S^{p-1}\right) \rightarrow\left(S^{p}, u\right)$. Choose a homotopy $G: I \times\left(D^{p}, S^{p-1}\right) \rightarrow\left(S_{+}^{p+1}, u\right)$ such that $G_{0}=f$ and $G_{1}\left(D^{p}\right)=u$ and define a proper isotopy $F$ of $D^{p} \times S^{0}$ in $D^{p} \times S^{p+1}$ by

$$
F_{t}(x, i):=\left(x, i \cdot G_{t}(x)\right) \quad\left(0 \leq t \leq 1, x \in D^{p}, i= \pm 1\right) .
$$

Then $F_{0} \in \Omega_{p} E\left(S^{0}, S^{p}\right), F_{1}=\mathrm{id}$, and $F$ is fixed on $S^{p-1} \times S^{0}$. Clearly

$$
\begin{aligned}
\mu(F) & =\operatorname{sc}\left(I \times D^{p} \times a, F\left(I \times D^{p} \times S^{0}\right) ; I \times D^{p} \times S^{p+1}\right) \\
& =\operatorname{sc}\left(I \times D^{p} \times a, F\left(I \times D^{p} \times 1\right) ; I \times D^{p} \times S_{+}^{p+1}\right) \\
& =\text { local degree of } G \text { over the point } a \\
& =\text { degree of } G:\left(I \times D^{p}, \partial\left(I \times D^{p}\right)\right) \rightarrow\left(S_{+}^{p+1}, S^{p-1}\right) \\
& =\operatorname{deg} f=1 .
\end{aligned}
$$

This proves that $\lambda: \pi_{p} E\left(S^{0}, S^{p}\right) \rightarrow \mathbb{Z}_{d}$ is surjective. 
For the induction step it suffices to prove that the following diagram is commutative:

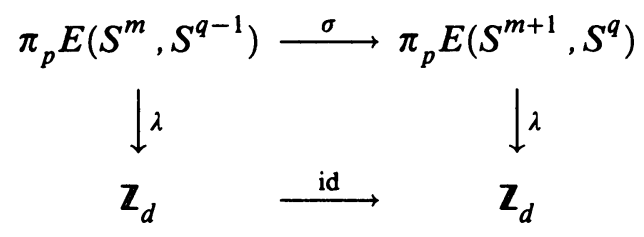

The proof of this is fairly straightforward. Take any $e \in \Omega_{p} E\left(S^{m}, S^{q-1}\right)$. Choose a proper isotopy $F$ of $D^{p} \times S^{m}$ in $D^{p} \times S^{q}$ (rel $\partial$ ) such that $F_{0}=e$ and $F_{1}=$ id (so that $\lambda[e]=\mu(F) \bmod d$ ). Represent $\sigma[e]$ by an embedding $e^{\prime} \in \Omega_{p} E\left(S^{m+1}, S^{q}\right)$ which agrees with $(\mathrm{id} \times \psi) e(\mathrm{id} \times \psi)^{-1}$ on $D^{p} \times\left(0 \times S^{m}\right)$ and embeds $D^{p} \times S_{1, i}^{m+1}$ properly into $D^{p} \times S_{1, i}^{q}(i=+,-$; see Lemma 1.4). There exists a proper concordance $F^{\prime}$ of $D^{p} \times S^{m+1}$ in $D^{p} \times S^{q+1}$ such that

$$
\begin{gathered}
F_{0}^{\prime}=e^{\prime}, \quad F_{1}^{\prime}=\mathrm{id}, \quad F^{\prime} \mid I \times S^{p-1} \times S^{m+1}=\mathrm{id}, \\
F^{\prime} \mid\left(I \times D^{p}\right) \times\left(0 \times S^{m}\right)=(\mathrm{id} \times \psi) F(\mathrm{id} \times \psi)^{-1}
\end{gathered}
$$

and

$$
\left(I \times D^{p} \times 0 \times S^{q}\right) \cap F^{\prime}\left(I \times D^{p} \times S^{m+1}\right)=(\mathrm{id} \times \psi) F\left(I \times D^{p} \times S^{m}\right)
$$

(so that $\lambda\left[e^{\prime}\right]=\mu\left(F^{\prime}\right) \bmod d$ ); in fact, the first four conditions for $F^{\prime}$ define an embedding of $\partial\left(I \times D^{p} \times S_{1, i}^{m+1}\right)$ into $\partial\left(I \times D^{p} \times S_{1, i}^{q+1}\right) \quad(i=+,-)$, which can then be extended to a proper embedding of $I \times D^{p} \times S_{1, i}^{m+1}$ into $I \times D^{p} \times S_{1, i}^{q+1}$.

Let $a:=\left(0^{q}, 1\right) \in S^{q}$ and $a^{\prime}:=\left(0^{q+1}, 1\right)=\psi(a) \in S^{q+1}$. Then

$$
\begin{aligned}
\mu\left(F^{\prime}\right) & =\operatorname{sc}\left(I \times D^{p} \times a^{\prime}, F^{\prime}\left(I \times D^{p} \times S^{m+1}\right) ; I \times D^{p} \times S^{q+1}\right) \\
& =\operatorname{sc}\left(I \times D^{p} \times a^{\prime},(\mathrm{id} \times \psi) F\left(I \times D^{p} \times S^{m}\right) ; I \times D^{p} \times 0 \times S^{q}\right) \\
& =\operatorname{sc}\left(I \times D^{p} \times a, F\left(I \times D^{p} \times S^{m}\right) ; I \times D^{p} \times S^{q}\right) \\
& =\mu(F),
\end{aligned}
$$

and Proposition 1.17 is proved.

We shall conclude this lengthy section with an extension of the function $s$. Let $p \geq 1$ and $m \geq 1$. Let $A$ be an integral relative $p$-cycle in $\left(D^{p} \times\right.$ $\left.S^{m+p}, S^{p-1} \times S^{m+p}\right)$ (e.g. a compact, oriented, proper $p$-submanifold of $D^{p} \times S^{m+p}$ ) such that the support of $\partial A$ misses $S^{p-1} \times S^{m}$. For each $e$ in $\Omega_{p} E\left(S^{m}, S^{m+p}\right)$ let

$$
s(A, e):=\operatorname{sc}\left(A, e\left(D^{p} \times S^{m}\right) ; D^{p} \times S^{m+p}\right) .
$$

Again, $s(A, e)$ does not really depend on $e$ but only on $[e]$. However, it is not independent of $A$. In fact we have the following lemma. 
1.18. Lemma. Suppose that $\partial A$ represents $n$ times the "positive" generator of $H_{p-1}\left(S^{p-1} \times S^{m+p}\right)$. Then $s(A, e)=n \cdot s(e)+s(A$, id) for each $e \in$ $\Omega_{p} E\left(S^{m}, S^{m+p}\right)$.

Proof. Take any $a \in S^{m+p}-S^{m}$. By hypothesis there exists an integral $p$ chain $C$ in $S^{p-1} \times S^{m+p}$ such that $\partial C$ is the formal linear combination $\partial A-$ $n\left(S^{p-1} \times a\right)$. Since $m \geq 1$ the $p$-cycle $A-C-n\left(S^{p-1} \times a\right)$ is homologous to zero in $D^{p} \times S^{m+p}$ and therefore it has zero intersection number with $e\left(D^{p} \times S^{m}\right)$ for each $e \in \Omega_{p} E\left(S^{m}, S^{m+p}\right)$. Hence

$$
\begin{aligned}
s(A, e) & =s\left(n\left(D^{p} \times a\right), e\right)+\operatorname{sc}\left(C, e\left(D^{p} \times S^{m}\right) ; D^{p} \times S^{m+p}\right) \\
& =n \cdot s(e)+(-1)^{p} \operatorname{sc}\left(C, S^{p-1} \times S^{m} ; S^{p-1} \times S^{m+p}\right) .
\end{aligned}
$$

Inserting $e=$ id here we find that the second term in the last line above is equal to $s(A, \mathrm{id})$, whence we obtain the formula asserted in the lemma.

\section{Puncturing A Closed SUbManifold of $\mathbf{R}^{q}$}

Throughout this section let $M$ denote an arbitrary closed, $k$-connected $m$ dimensional submanifold of $\mathbb{R}^{q}$, where $1 \leq k \leq m-4$ and $q:=2 m-k-1$. Choose an $m$-disk $\Delta \subset M$ and let $M_{0}:=M-\operatorname{int} \Delta$.

Recall that $\mathscr{E}\left(X, \mathbb{R}^{q}\right)$ denotes the set of all embeddings of a polyhedron $X$ into $\mathbb{R}^{q}$ and that $\mathscr{I}\left(X, \mathbb{R}^{q}\right)$ is the set of isotopy classes of such embeddings. In this section we shall describe the set $\mathscr{I}\left(M_{0}, \mathbf{R}^{q}\right)$ and find a relation between $\mathscr{I}\left(M_{0}, \mathbb{R}^{q}\right)$ and $\mathscr{I}\left(M, \mathbb{R}^{q}\right)$. The following are our results:

\subsection{Theorem. There exists a canonical bijection}

$$
\gamma: \mathscr{I}\left(M_{0}, \mathbb{R}^{q}\right) \succ H_{k+1}\left(M ; \pi_{m-k-1} E\left(D^{k+1}, D^{m}\right)\right) .
$$

Recall that $\pi_{m-k-1} E\left(D^{k+1}, D^{m}\right)$ is $\mathbb{Z}$ if $m-k$ is odd and is $\mathbb{Z}_{2}$ if $m-k$ is even (Lemma 1.2 and Theorem 1.15).

2.2. Addendum. If $P$ is a spine of $M_{0}$, then $2 \gamma$ is, up to sign, the composite of the function

$$
\begin{gathered}
\mathscr{I}\left(M_{0}, \mathbb{R}^{q}\right) \rightarrow H^{m-k-1}(P ;-), \\
\left.e \mapsto e^{*} \text { (Alexander dual of }\left[e\left(\partial M_{0}\right)\right] \in H_{m-1}\left(\mathbf{R}^{q}-e(P) ;-\right)\right),
\end{gathered}
$$

and the sequence of isomorphisms

$$
\begin{aligned}
H^{m-k-1}(P ;-) & \approx H^{m-k-1}\left(M_{0} ;-\right) \approx H_{k+1}\left(M_{0}, \partial M_{0} ;-\right) \\
& \approx H_{k+1}(M, \Delta ;-) \approx H_{k+1}(M ;-)
\end{aligned}
$$

(coefficients $\pi_{m-k-1} E\left(D^{k+1}, D^{m}\right)$ everywhere).

Let $r: \mathscr{E}\left(M, \mathbb{R}^{q}\right) \rightarrow \mathscr{E}\left(M_{0}, \mathbb{R}^{q}\right)$ be the restriction map and let $r_{*}: \mathscr{I}\left(M, \mathbf{R}^{q}\right)$ $\rightarrow \mathscr{I}\left(M_{0}, \mathbb{R}^{q}\right)$ be the map induced by $r$. 
2.4. Theorem. The composite $\gamma r_{*}$ maps $\mathscr{I}\left(M, \mathbf{R}^{q}\right)$ onto the 2-torsion subgroup of $H_{k+1}\left(M ; \pi_{m-k-1} E\left(D^{k+1}, D^{m}\right)\right.$ ) (the elements of order $\left.\leq 2\right)$. In particular, $r_{*}$ is surjective if $m-k$ is even.

The second assertion of this theorem follows from the first one since

$$
\pi_{m-k-1} E\left(D^{k+1}, D^{m}\right)=\mathbb{Z}_{2}
$$

if $m-k$ is even.

2.5. Corollary. If $m-k$ is odd and $H_{k+1}(M)$ has no 2-torsion or if $m-k$ is even and $H_{k+1}\left(M ; \mathbb{Z}_{2}\right)=0$, then an arbitrary embedding $e: M \rightarrow \mathbf{R}^{q}$ is isotopic (inside $\mathbf{R}^{q}$ ) to an embedding $e^{\prime}$ such that $e^{\prime} \mid M_{0}=\mathrm{id}$ and hence $e$ is isotopic inside $\mathbf{R}^{q+1}$ to the inclusion.

Proof. The stated conditions imply, by $2.4,1.2$, and 1.15 , that $r_{*}$ is constant. This gives the first assertion of 2.5. The second assertion is proved by pushing the interiors of the $m$-disks $\Delta$ and $e^{\prime}(\Delta)$ slightly into int $R_{+}^{q+1}$ and then deforming (rel $\partial$ ) the latter disk onto the former inside $\mathbb{R}_{+}^{q+1}$.

Throughout the rest of this section we shall write $m-k-1=: p$. Note that our hypothesis that $k \geq 1$ implies that $q \geq 2 p+2$. According to Wall [15, Theorem 3], $M_{0}$ has a $p$-dimensional spine $P \subset$ int $M_{0}$. We choose and fix such a $P$ for the rest of this section.

Proof of Theorem 2.1. Denote by Imm the set of all immersions of $M_{0}$ into $\mathbf{R}^{q}$ and by [Imm] the set of "immersion concordance classes" of such immersions; that is, immersions $i_{0}, i_{1}: M_{0} \rightarrow \mathbf{R}^{q}$ represent the same element of [Imm] if and only if there exists an "immersion concordance" between $i_{0}$ and $i_{1}$, i.e. an immersion $F: I \times M_{0} \rightarrow I \times \mathbf{R}^{q}$ which maps $t \times M_{0}$ into $t \times \mathbb{R}^{q}$ by $i_{t}$ $(t=0,1)$ and sends (int $I) \times M_{0}$ into (int $\left.I\right) \times \mathbf{R}^{q}$.

It is easy to see that the inclusion $\mathscr{E}\left(M_{0}, \mathbf{R}^{q}\right) \hookrightarrow$ Imm induces a bijection $\mathscr{I}\left(M_{0}, \mathbf{R}^{q}\right) \nrightarrow[$ Imm]. Indeed, by a general position argument an arbitrary immersion $i: M_{0} \rightarrow \mathbb{R}^{q}$ is regularly homotopic (hence immersion-concordant) to an immersion $i^{\prime}$ which is an embedding on $P$ and hence an embedding on a small regular neighborhood $N$ of $P$. It we take an isotopy in $M_{0}$ which shrinks $M_{0}$ to $N$ and compose all stages of this isotopy with $i^{\prime}$, we obtain a regular homotopy from $i^{\prime}$ to an embedding. By a similar reference to general position and uniqueness of regular neighborhoods we can transform an arbitrary immersion concordance between two embeddings $e_{0}, e_{1}: M_{0} \rightarrow \mathbb{R}^{q}$ into a concordance, and consequently into an isotopy, between $e_{0}$ and $e_{1}$.

If $i: M_{0} \rightarrow \mathbb{R}^{q}$ is an immersion, then by G. P. Scott's block bundle variant [12] of the Haefliger-Poenaru PL immersion theory [3], the only obstructions to immersion concordance between $i$ and the inclusion lie in the groups

$$
H^{s}\left(M_{0} ;\left\{\pi_{s}\left(\tilde{V}_{q, m}\right)\right\}\right)=H^{s}\left(P ;\left\{\pi_{s} E\left(D^{m}, D^{q}\right)\right\}\right)
$$


(a little more about this will be said in the proof of Proposition 2.8). Since $\pi_{s} E\left(D^{m}, D^{q}\right)=0$ for $s<p$, by Theorem 1.9 (note that $q-m=p \geq 3$ ), and since $H^{s}(P ;-)=0$ for $s>p$, the only obstruction lies in

$$
H^{p}\left(P ; \pi_{p} E\left(D^{m}, D^{q}\right)\right) \approx H^{p}\left(P ; \pi_{p} E\left(D^{m-p}, D^{m}\right)\right)
$$

(there is no twist in the coefficients since $M$ is orientable); the latter isomorphism is induced by a higher suspension isomorphism of the coefficient groups (see Proposition 1.5). By standard obstruction theory (our obstruction to immersion concordance is just the usual obstruction to homotopy between two sections of a Kan fibration), assigning to each immersion $i$ the corresponding obstruction element in the group $H^{p}\left(P ; \pi_{p} E\left(D^{m-p}, D^{m}\right)\right)$ determines a bijection of [Imm] onto this group. In this way we get a bijection

$$
\zeta: \mathscr{I}\left(M_{0}, \mathbf{R}^{q}\right) \rightarrow H^{p}\left(P ; \pi_{p} E\left(D^{m-p}, D^{m}\right)\right) .
$$

We denote by $\gamma$ the composite of $\zeta$ with the isomorphisms (2.3). Theorem 2.1 is proved.

From now on we shall write $\pi_{p} E\left(D^{k+1}, D^{m}\right)=: \pi_{p}$. In our proof of Addendum 2.2 and Theorem 2.4, the first step will be representing the bijection $\zeta$ of (2.6) by a simple, geometrically defined function giving explicit simplicial cocycles.

Choose a triangulation $\left(T, T_{M}, T_{P}\right)$ of the triple $\left(\mathbb{R}^{q}, M, P\right)$ and let $T^{\prime \prime}$, $T_{M}^{\prime \prime}, T_{P}^{\prime \prime}$ be the second barycentric subdivisions of the complexes $T, T_{M}, T_{P}$. Then $N\left(P, T_{M}^{\prime \prime}\right)$, the simplicial neighborhood of $P$ in $T_{M}^{\prime \prime}$, is a regular neighborhood of $P$ in $M$ just as $M_{0}$ is, and by uniqueness of regular neighborhoods we can replace $M_{0}$ by $N:=N\left(P, T_{M}^{\prime \prime}\right)$ in our proofs. Let $W:=N\left(P, T^{\prime \prime}\right)$. Then the pair $(W, N)$ is a regular neighborhood of $P$ in $\left(\mathbf{R}^{q}, M\right)$.

The pair $(W, N)$ has a canonical handle decomposition: the handles are the stars in $\left(T^{\prime \prime}, T_{M}^{\prime \prime}\right)$ of the barycenters of the simplices of $T_{P}$. We shall use only the handles of index $p$. So let $\sigma_{1}, \sigma_{2}, \ldots$ be the $p$-simplices of $T_{P}$ and for each $i$ let $b_{i}$ be the barycenter of $\sigma_{i}$ and

$$
A_{i}:=N\left(b_{i}, T_{M}^{\prime \prime}\right), \quad B_{i}:=N\left(b_{i}, T^{\prime \prime}\right), \quad C_{i}:=N\left(b_{i}, T_{P}^{\prime \prime}\right) .
$$

Denote by $P_{0}$ the underlying space of the $(p-1)$-skeleton of $T_{P}$. Each pair $\left(B_{i}, A_{i}\right)$ is a $p$-handle pair of $(W, N)$; it is attached to the pair

$$
W_{0}:=N\left(P_{0}, T^{\prime \prime}\right), \quad N_{0}:=N\left(P_{0}, T_{M}^{\prime \prime}\right)
$$

and has core disk $C_{i}$.

Let $D:=M-$ int $N$. Denote by $\mathscr{E}_{0}(N, W)$ the set of all embeddings $N \rightarrow W$ that are identity on $P \cup N_{0}$ and embed each handle $A_{i}$ of $N$ properly into the handle $B_{i}$ of $W$. Let $\mathscr{E}_{0}\left(M, \mathbf{R}^{q}\right)$ be the set of all embeddings $e: M \rightarrow \mathbf{R}^{q}$ such that $e \mid N$ belongs to $\mathscr{E}_{0}(N, W)$ and $e(D)$ is properly embedded in $\mathbf{R}^{q}-$ int $W$. 
2.7. Lemma. The composites $\mathscr{E}_{0}(N, W) \hookrightarrow \mathscr{E}\left(N, \mathbf{R}^{q}\right) \rightarrow \mathscr{I}\left(N, \mathbf{R}^{q}\right)$ and

$$
\mathscr{E}_{0}\left(M, \mathbf{R}^{q}\right) \hookrightarrow \mathscr{E}\left(M, \mathbf{R}^{q}\right) \rightarrow \mathscr{I}\left(M, \mathbf{R}^{q}\right)
$$

are surjections.

Proof. This follows from standard theorems of PL topology. Take e.g. an embedding $e: M \rightarrow \mathbf{R}^{q}$. Since the pair $\left(N_{0}, \partial N_{0}\right)$ is $(k+1=2 m-q)$-connected, $N_{0}$ unknots in $\mathbf{R}^{q}$ [4, Theorem 10.3, p. 201], so $e$ is isotopic to an embedding $e_{1}$ such that $e_{1} \mid N_{0}=\mathrm{id}$. In the second step we use uniqueness of relative regular neighborhoods to find an embedding $e_{2}$, isotopic to $e_{1}$, such that $e_{2} \mid N_{0}=$ id and $e_{2}\left(M-N_{0}\right) \subset \mathbf{R}^{q}-W_{0}$. In the third step we use $p$-connectedness of $\mathbf{R}^{q}-$ int $W_{0}$ and the fact that $q \geq 2 p+2$ to construct an isotopy of $\mathbf{R}^{q}$ which is fixed on $W_{0}$ and moves $e_{2} \mid C_{i}$ to the inclusion for each $i$. The fourth step is similar to the second one and produces an embedding $e_{4} \in \mathscr{E}_{0}\left(M, \mathbf{R}^{q}\right)$, isotopic to $e$. The proof that $\mathscr{E}_{0}(N, W)$ maps onto $\mathscr{I}\left(N, \mathbf{R}^{q}\right)$ is almost equal.

Now we come to our intermediate goal, i.e. a geometric interpretation of the bijection $\zeta$ of (2.6). Orient $M$ and all $p$-simplices of $T_{P}$. For each $p$-simplex $\sigma_{i} \in T_{P}$ choose a homeomorphism

$$
g_{i}:\left(D^{q}, D^{m}, D^{p}, S^{p-1} \times D^{m}\right) \rightarrow\left(B_{i}, A_{i}, C_{i}, B_{i} \cap W_{0}\right)
$$

which is orientation preserving on the first three terms of the quadruple. For an arbitrary $e \in \mathscr{E}_{0}(N, W)$ let $z(e) \in C^{p}\left(T_{P} ; \pi_{p}\right)=Z^{p}\left(T_{P} ; \pi_{p}\right)$ be the simplicial cocycle which assigns to each $p$-simplex $\sigma_{i} \in T_{P}$ (with the chosen orientation) the element of $\pi_{p}$ represented by the embedding $f_{i}:=g_{i}^{-1} e g_{i}: D^{m} \rightarrow D^{q}$ (which indeed belongs to $\Omega_{p} E\left(D^{k+1}, D^{m}\right)$ ).

2.8. Proposition. $\zeta([e])=[z](e):=[z(e)] \in H^{p}\left(P ; \pi_{p}\right)$ for each $e \in \mathscr{E}_{0}(N, W)$. Proof. Take an embedding $e \in \mathscr{E}_{0}(N, W)$ and choose a homotopy $F: I \times N \rightarrow$ $\mathbf{R}^{q}\left(\right.$ rel $\left.N_{0} \cup P\right)$ between $N \hookrightarrow \mathbf{R}^{q}$ and $e$. By a block bundle variant of Haefliger and Poenaru [3, p. 83] there is a Kan fibration over $I \times N$ (actually over a suitable partially ordered triangulation of $I \times N$ considered as a $\Delta$-set) with total space $\mathscr{R}_{F}$ consisting of "representations of $T N$ to $T R^{q}$ "; $\mathscr{R}_{F}$ has fibers isomorphic to the block PL Stiefel manifold $\tilde{V}_{q, m}[10$, p. 274] and is such that its sections correspond bijectively to those homotopies of representations of $T N$ in $T R^{q}$ which cover the map $F$ (see also Scott [12]). We omit the precise definition of $\mathscr{R}_{F}$.

Finding an immersion concordance between $N \hookrightarrow \mathbf{R}^{q}$ and $e$ is by Scott [12] equivalent to finding a homotopy between the representations $T N \hookrightarrow T \mathbf{R}^{q}$ and de: $T N \rightarrow T \mathrm{R}^{q}$, hence to constructing a section $S$ of $\mathscr{R}_{F}$ such that $S \mid 0 \times N$ corresponds to $T N \hookrightarrow T \mathbf{R}^{q}$ and $S \mid 1 \times N$ to de. Obviously it suffices to construct $S$ over $I \times\left(N_{0} \cup P\right)$. Since $e=$ id on $N_{0}$ we can take $S$ "constant" over $I \times N_{0}$. Thus the $p$ th suspension

$$
\left(\Sigma^{p}\right)_{*} \zeta[e] \in H^{p}\left(P ; \pi_{p} E\left(D^{m}, D^{q}\right)\right)
$$


(this is the cohomological obstruction introduced in the proof of Theorem 2.1) is the cohomology class of the obstruction to extending $S$ over the rest of $I \times\left(N_{0} \cup P\right)$, i.e. the cohomology class of the simplicial $p$-cocycle on $T_{P}$ which assigns to each $p$-simplex $\sigma_{i}$ the homotopy class in $\pi_{p}\left(\tilde{V}_{q, m}\right)$ determined by $S \mid \partial\left(I \times C_{i}\right)$ (where $S$ is given by de over $1 \times C_{i}$ and by the inclusion over the rest of $\left.\partial\left(I \times C_{i}\right)\right)$.

We shall now transfer this to the standard model using the homeomorphisms $g_{i}: D^{q} \rightarrow B_{i}$ and their differentials $d g_{i}: T D^{q} \rightarrow T B_{i}$. We shall thus consider the elements of $\pi_{p}\left(\tilde{V}_{q, m}\right)$ determined by $d g_{i}^{-1} d e d g_{i}=d f_{i}: T D^{m} \mid D^{p} \rightarrow$ $T D^{q} \mid D^{p}$. We can regard $T D^{m}$ and $T D^{q}$ as restrictions of $T \mathrm{R}^{m}$ and $T \mathrm{R}^{q}$, respectively, and it will be convenient for us to look at these not just over $D^{p}$ but over the whole $\mathbb{R}^{p}$; we can think of this $\mathbb{R}^{p}$ as the $p$-sphere $\partial\left(I \times D^{p}\right)$ with the point $0^{1+p}$ removed and of $D^{p}$ as $1 \times D^{p} \subset \partial\left(I \times D^{p}\right)$. In accordance with this we extend $f_{i}: D^{p} \times D^{m-p} \rightarrow D^{p} \times D^{m}$ to a proper embedding $\mathbf{R}^{p} \times D^{m-p} \rightarrow \mathbb{R}^{p} \times D^{q-p}$, again denoted by $f_{i}$, which is equal to the inclusion outside $D^{p} \times D^{m-p}$.

We shall henceforth assume that all $f_{i}$ are level preserving: $f_{i}(x, y)=$ $\left(x, \varphi_{i}(x, y)\right)$ for some $\varphi_{i}: \mathbb{R}^{p} \times D^{m-p} \rightarrow D^{m}$ (and $\varphi_{i}(x, y)=y$ if $x \in$ $\mathbb{R}^{p}$-int $\left.D^{p}\right)$; this is permissible by Lemma 1.16 and will be of great help in the sequel.

For $n=m, q$ consider the product block bundle $\mathbb{R}^{n} \times D^{n}$ over (some suitable cell decomposition of ) $\mathbb{R}^{n}$ and the self-homeomorphism $\psi_{n}$ of $\mathbf{R}^{n} \times \mathbf{R}^{n}$ defined by $\psi_{n}(x, y):=(x, x+y)$. The space $\psi_{n}\left(\mathbb{R}^{n} \times D^{n}\right)$ will be our model for the tangent block bundle $T \mathbb{R}^{n}$ (recall that the tangent block bundle of a manifold $Q$ is not a specific block bundle but an isomorphism class of block bundles over various cell complexes on $Q$, with total space a regular neighborhood of the diagonal in $Q \times Q$ and with "zero section" $x \mapsto(x, x))$. Actually we shall replace this $T \mathbb{R}^{n}$ back by $\mathbb{R}^{n} \times D^{n}$ and, accordingly, $T \mathbf{R}^{n} \mid \mathbb{R}^{p}$ by $\mathbf{R}^{p} \times D^{n}$ (we ought to write $\mathbb{R}^{p} \times 0^{n-p} \times D^{n}$ here but we suppress the $0^{n-p}$ factor). Hence each differential $d f_{i}: T \mathbb{R}^{m}\left|\mathbb{R}^{p} \rightarrow T \mathbb{R}^{q}\right| \mathbb{R}^{p}$, which is by definition a restriction of $f_{i} \times f_{i}$, will be replaced by the proper embedding

$$
F_{i}:=\psi_{q}^{-1}\left(f_{i} \times f_{i}\right) \psi_{m}: \mathbb{R}^{p} \times\left(D^{p} \times D^{m-p}\right) \rightarrow \mathbb{R}^{p} \times\left(D^{p} \times D^{m}\right),
$$

which is given by $F_{i}(\xi, x, y)=\left(\xi, x, \varphi_{i}(\xi+x, y)\right)$.

We see now that $\left(\Sigma^{p}\right)_{*} \zeta([e])$ is represented by the $p$-cocycle on $T_{P}$ that sends each $p$-simplex $\sigma_{i}$ to the proper isotopy class of $F_{i}: 2 D^{p} \times D^{m} \rightarrow$ $2 D^{p} \times D^{q}$. (We must take the cube $2 D^{p}:=[-2,2]^{p}$ rather than $D^{p}$ as our "parameter space" here in order to have the identity along the boundary. Clearly this replacing $D^{p}$ by $2 D^{p}$ can do no harm. We could have preserved our standard $D^{p}$ at the expense of introducing some reparametrizations and other minor complications earlier in the construction.) By definition of suspension, $\left(\Sigma^{p}\right)_{*}[z(e)]$ is represented by the $p$-cocycle which sends each $\sigma_{i}$ to the proper isotopy class of the embedding $(\xi, x, y) \mapsto\left(\xi, x, \varphi_{i}(\xi, y)\right)$. The latter 
is obviously properly isotopic $\left(\operatorname{rel} \partial\left(2 D^{p}\right) \times D^{m}\right)$ to $F_{i}$, which concludes the proof of Proposition 2.8.

Remarks. 1. In our proofs of Theorem 2.1 and Proposition 2.8 we have never really used the fact that $M_{0}$ is a punctured closed, $k$-connected manifold. Therefore the same proofs demonstrate the existence of the bijection $\zeta$ and validity of Proposition 2.8 if $M_{0} \subset \mathbf{R}^{q}$ is an arbitrary compact, connected $m$ manifold having a $p$-dimensional spine $P$ where $3 \leq p=q-m \leq m-2$; however, if $M_{0}$ is nonorientable, we must twist the coefficients of the group $H^{p}\left(P ; \pi_{p}\right)$ along with the orientation of the normal block bundle of $M_{0}$ in $\mathbb{R}^{q}$.

2. One can construct a direct and elementary, but fairly long and complicated, proof that the function $[z]: \mathscr{E}_{0}(N, W) \rightarrow H^{p}\left(P ; \pi_{p}\right)$ induces a bijection $\mathscr{I}\left(N, \mathbf{R}^{q}\right) \rightarrow H^{p}\left(P ; \pi_{p}\right)$ (also in the more general situation of Remark 1 above). In this way one can avoid immersion theory and Proposition 2.8.

We shall now define two other functions from $\mathscr{E}_{0}(N, W)$ to the $p$ th cohomology of $P$. The definition of the first one will be based on the cone over $P$. Denote this cone by $c P$ and for each subpolyhedron $S \subset P$ let $c S$ be the subcone of $c P$ with base $S$.

2.9. Lemma. The inclusion $P \hookrightarrow \mathbf{R}^{q}$ can be extended to an embedding of $c P$ in $\mathbf{R}^{q}$.

Proof. Choose a general position map $f: c P \rightarrow \mathbf{R}^{q}$ such that $f \mid P=$ id. The dimension of the singular set of $f$ is at most 0 . Therefore there exists a "horizontal section" $P^{\prime}$ of $c P$ so close to the vertex of the cone that the portion of $c P$ above $P^{\prime}$ is embedded by $f$. This means that the obvious homeomorphism $P \rightarrow f\left(P^{\prime}\right)$ can be extended to an embedding $c P \rightarrow \mathbb{R}^{q}$. Since $P$ unknots in $\mathbf{R}^{q}$ the lemma follows.

Choose, and fix, an embedding $c P \rightarrow \mathbf{R}^{q}$ (which is identity on $P$ ) such that the images of both $c P$ and $c P_{0}$ are in general position with $M$. To simplify the notation we shall denote the image of this embedding simply by $c P$. Our general position requirement means that

$$
\operatorname{dim}((c P-P) \cap M) \leq 1 \text { and } \operatorname{dim}\left(\left(c P_{0}-P\right) \cap M\right) \leq 0 .
$$

Assume that the triangulation $T$ of $\mathrm{R}^{q}$ was chosen so that appropriate subcomplexes of $T$ triangulate $c P$ and $c P_{0}$. Then $c P \cap W$ is a collar on $P$ and, for each $p$-simplex $\sigma_{i} \in T_{P}, c \sigma_{i} \cap W$ is a collar on $\sigma_{i}$. For each $i$ let $\sigma_{i}^{\prime}:=c \sigma_{i} \cap \partial W$ and $C_{i}^{\prime}:=\sigma_{i}^{\prime} \cap B_{i}$. Note that $\sigma_{i}^{\prime}$ and $C_{i}^{\prime}$ are $p$-disks and

$$
\partial \sigma_{i}^{\prime} \cap \partial N=c P_{0} \cap \partial N=\varnothing, \quad \partial C_{i}^{\prime} \cap \partial N=\varnothing .
$$

Define a function $u: \mathscr{E}_{0}(N, W) \rightarrow C^{p}\left(T_{P}\right)=Z^{p}\left(T_{P}\right)$ as follows: for each $e \in \mathscr{E}_{0}(N, W)$ and each (oriented) $p$-simplex $\sigma_{i} \in T_{P}$ let

$$
\left\langle\sigma_{i}, u(e)\right\rangle:=\operatorname{sc}\left(c \sigma_{i}, e(\partial N) ; \mathbf{R}^{q}\right)=\operatorname{sc}\left(\sigma_{i}^{\prime}, e(\partial N) ; \partial W\right),
$$


where $c \sigma_{i}$ is oriented incoherently with $\sigma_{i}$ and then $\sigma_{i}^{\prime}$ coherently with $c \sigma_{i} \cap W$ (since $e \in \mathscr{E}_{0}(N, W)$, the intersection numbers are well defined by (2.10)). Denote by $[u(e)]$ the cohomology class of $u(e)$ and define $[u]: \mathscr{E}_{0}(N, W) \rightarrow$ $H^{p}(P)$ by $e \mapsto[u(e)]$. Recall that $r: \mathscr{E}\left(M, \mathbf{R}^{q}\right) \rightarrow \mathscr{E}\left(N, \mathbf{R}^{q}\right)$ is the restriction map.

2.11. Lemma. $r\left(\mathscr{E}_{0}\left(M, \mathbf{R}^{q}\right)\right)=[u]^{-1}(0)$. For any $e \in \mathscr{E}_{0}(N, W),[u(e)]$ is up to sign the Alexander dual of $[e(\partial N)] \in H_{m-1}\left(\mathbf{R}^{q}-P\right)$.

Proof. We first prove the second assertion. The usual construction of the Alexander dual (for a certain sign convention) is the following: let $f: D \rightarrow \mathbb{R}^{q}$ be an extension of $e \mid \partial N$ such that $f(D)$ misses the $(p-1)$-skeleton of $c P$; then the dual of $[e(\partial N)]$ is the cohomology class of the cocycle $a \in Z^{p}\left(T_{P}\right)$ given by $\left\langle\sigma_{i}, a\right\rangle:=\operatorname{sc}\left(\sigma_{i}, f(D) ; \mathbf{R}^{q}\right)$. Now

$$
\begin{aligned}
\left\langle\sigma_{i}, u(e)\right\rangle & =-\operatorname{sc}\left(c \sigma_{i}, f(\partial D) ; \mathbf{R}^{q}\right)=(-1)^{p} \operatorname{sc}\left(\partial\left(c \sigma_{i}\right), f(D) ; \mathbf{R}^{q}\right) \\
& =(-1)^{p}\left(\operatorname{sc}\left(\sigma_{i}, f(D) ; \mathbb{R}^{q}\right)-\operatorname{sc}\left(c\left(\partial \sigma_{i}\right), f(D) ; \mathbf{R}^{q}\right)\right) \\
& =(-1)^{p}\left\langle\sigma_{i}, a-\delta b\right\rangle,
\end{aligned}
$$

where $b \in C^{p-1}\left(T_{P}\right)$ assigns to each oriented $(p-1)$-simplex $\tau \in T_{P}$ the number $\operatorname{sc}\left(c \tau, f(D) ; \mathbf{R}^{q}\right)$. Thus $[u(e)]= \pm[a]$ as asserted.

The first assertion of Lemma 2.11 is now easy to prove. If $e \in \mathscr{E}_{0}\left(M, \mathbf{R}^{q}\right)$, then $e(\partial N)$ bounds $e(D) \subset \mathbf{R}^{q}-P$ and hence $[u(e \mid N)]=0$. Conversely, if $e \in \mathscr{E}_{0}(N, W)$ is such that $[u(e)]=0$, then $e(\partial N)$ bounds homologically in $\mathbf{R}^{q}-P$, and therefore, as $\mathbf{R}^{q}-P$ is $(m-2)$-connected, $e(\partial N)$ bounds a singular $m$-disk in $\mathbf{R}^{p}-P$. Now Irwin's Embedding Theorem implies that $e(\partial N)$ bounds a nonsingular $m$-disk in $\mathbf{R}^{q}-$ int $W$, i.e. $e \in r\left(\mathscr{E}_{0}\left(M, \mathbf{R}^{q}\right)\right)$.

Recall the homeomorphisms $g_{i}: D^{q} \rightarrow B_{i}$ used in the definition of the cocyles $z(e)$. Pick a point $y \in S^{m-1}-S^{k}$ and let $K_{i}:=g_{i}\left(D^{p} \times y\right)$ for each $p$-simplex $\sigma_{i} \in T_{P}$. For each $e \in \mathscr{E}_{0}(N, W)$ define $v(e) \in Z^{p}\left(T_{P}\right)$ by

$$
\left\langle\sigma_{i}, v(e)\right\rangle:=\operatorname{sc}\left(K_{i}, e(\partial N) ; \partial W\right)
$$

(orientation in $K_{i}$ is induced by $g_{i}$ ). Let $[v]: \mathscr{E}_{0}(N, W) \rightarrow H^{p}(P)$ be given by $[v](e):=[v(e)]$.

2.12. Lemma. $[v]=[u]$.

Proof. Take any $e \in \mathscr{E}_{0}(N, W)$ and any $p$-simplex $\sigma_{i} \in T_{P}$. By (2.10) we can write

$$
\begin{aligned}
\left\langle\sigma_{i}, u(e)\right\rangle & =\operatorname{sc}\left(C_{i}^{\prime}, e(\partial N) ; \partial W\right)+\operatorname{sc}\left(\sigma_{i}^{\prime}-\operatorname{int} C_{i}^{\prime}, e(\partial N) ; \partial W\right) \\
& =\operatorname{sc}\left(C_{i}^{\prime}, e(\partial N) ; \partial W\right)+\operatorname{sc}\left(\sigma_{i}^{\prime}-\operatorname{int} C_{i}^{\prime}, \partial N ; \partial W\right),
\end{aligned}
$$

and it follows from Lemma 1.18 that

$$
\begin{aligned}
\operatorname{sc}\left(C_{i}^{\prime}, e(\partial N) ; \partial W\right) & =\operatorname{sc}\left(K_{i}, e(\partial N) ; \partial W\right)+\operatorname{sc}\left(C_{i}^{\prime}, \partial N ; \partial W\right) \\
& =\left\langle\sigma_{i}, v(e)\right\rangle+\operatorname{sc}\left(C_{i}^{\prime}, \partial N ; \partial W\right) .
\end{aligned}
$$


Hence

$$
\begin{aligned}
\left\langle\sigma_{i}, u(e)\right\rangle & =\left\langle\sigma_{i}, v(e)\right\rangle+\operatorname{sc}\left(\sigma_{i}^{\prime}, \partial N ; \partial W\right) \\
& =\left\langle\sigma_{i}, v(e)\right\rangle+\left\langle\sigma_{i}, u(\mathrm{id})\right\rangle .
\end{aligned}
$$

Thus $u(e)=v(e)+u(\mathrm{id})$. Since $[u(\mathrm{id})]=0$, by Lemma 2.11 , it follows that $[u(e)]=[v(e)]$.

Proof of Addendum 2.2 and Theorem 2.4. Recall that we have replaced $M_{0}$ by $N$. If $m-k$ is even ( $p$ odd), it follows from Corollary 1.14 that $[v]=0$. Then Lemmas 2.12 and 2.11 imply that $\mathscr{E}_{0}(N, W)$ lies in the image of $r$, hence (by Lemma 2.7) all of $\mathscr{I}\left(N, \mathbf{R}^{q}\right)$ is in the image of $r_{*}$. For odd $p$, Addendum 2.2 is a trivial consequence of Theorem 2.4 (since $\pi_{p}=Z_{2}$ ).

Suppose that $m-k$ is odd ( $p$ even). Note that according to our definitions (see 1.2 and (1.10))

$$
\left\langle\sigma_{i}, v(e)\right\rangle=s \rho\left(\left\langle\sigma_{i}, z(e)\right\rangle\right)
$$

for each embedding $e \in \mathscr{E}_{0}(N, W)$ and each $p$-simplex $\sigma_{i} \in T_{P}$. This equality, Lemma 2.12, and Proposition 2.8 imply commutativity of the following diagram, in which 2 denotes multiplication by 2 and $(s \rho / 2)_{*}$ is the isomorphism induced by the coefficient isomorphism $\frac{1}{2} s \rho: \pi_{p} E\left(D^{m-p}, D^{m}\right) \rightarrow \mathbf{Z}$ (see Theorem 1.15):

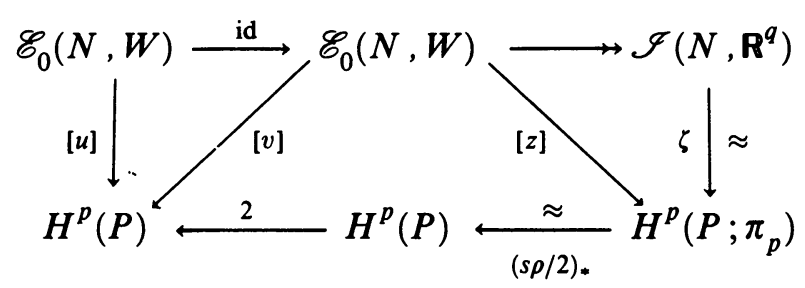

Lemmas 2.7 and 2.11 now obviously imply Addendum 2.2 and Theorem 2.4 for even $p$.

\section{Deforming A SUBMANIFOld of $\mathbf{R}^{q+1}$ INTO $\mathbf{R}^{q}$}

As in $\S 2$, let $M$ denote an arbitrary closed, $k$-connected $m$-dimensional submanifold of $\mathbf{R}^{q}$, where $1 \leq k \leq m-4$ and $q:=2 m-k-1$; let $\Delta$ be an $m$-disk in $M$ and $M_{0}:=M-$ int $\Delta$. The symbols $\mathscr{E}\left(M, \mathbf{R}^{q}\right)$ (for the set of embeddings $M \rightarrow \mathbf{R}^{q}$ ) and $\mathscr{I}\left(M, \mathbf{R}^{q}\right)$ (for the set of isotopy classes of such embeddings) will now be shortened to $\mathscr{E}^{q}$ and $\mathscr{I}^{q}$, respectively. Analogously we define $\mathscr{E}^{q+1}$ and $\mathscr{I}^{q+1}$. Our aim in this section is to describe the image of the function $\mathscr{I}^{q} \rightarrow \mathscr{I}^{q+1}$ induced by the inclusion $\mathscr{E}^{q} \hookrightarrow \mathscr{E}^{q+1}$.

Write $d:=1+(-1)^{m-k}$ and $\mathbf{Z}_{d}:=\mathbf{Z} / d \mathbf{Z}$. In [14] we showed that there exists a canonical bijection $\delta: \mathscr{J}^{q+1} \rightarrow H_{k+1}\left(M ; \mathbb{Z}_{d}\right)$ (this $\delta$ is the function $\delta($ id , -$)$ of [14, Theorem 1.2]). Here we will prove the following theorem (for notation see $1.2,1.17,2.1$, and 2.4 ). 
3.1. Theorem. The following diagram commutes up to sign:

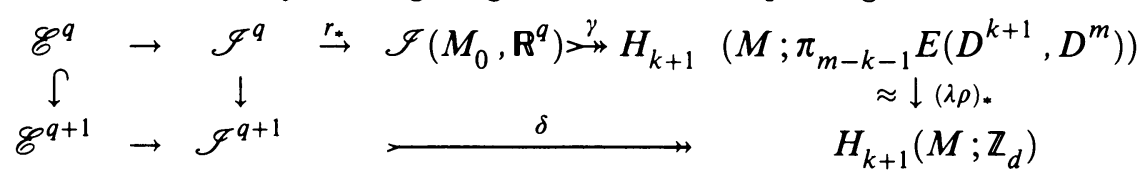

where $(\lambda \rho)_{*}$ is the isomorphism induced by the coefficient isomorphism

$$
\lambda \rho: \pi_{m-k-1} E\left(D^{k+1}, D^{m}\right) \rightarrow \mathbb{Z}_{d} .
$$

The following two corollaries, which subsume Corollary 2.5, are immediate consequences of Theorems 3.1, 2.1, and 2.4.

3.2. Corollary. The image of $\mathscr{I}^{q} \rightarrow \mathscr{I}^{q+1}$ is mapped by $\delta$ onto the 2-torsion subgroup of $H_{k+1}\left(M ; \mathbb{Z}_{d}\right)$. In particular, if $m-k$ is even, then every embedding $M \rightarrow \mathbf{R}^{q+1}$ is isotopic to an embedding $M \rightarrow \mathbf{R}^{q}$.

3.3. Corollary. Two embeddings of $M$ in $\mathbf{R}^{q}$ are isotopic in $\mathbb{R}^{q+1}$ if and only if their restrictions to $M_{0}$ are isotopic in $\mathbf{R}^{q}$.

In the proof of Theorem 3.1 we shall employ the following notation of $\S 2$ :

$p:=m-k-1$,

$P$ is a $p$-dimensional spine of $M_{0}$,

$\left(T, T_{M}, T_{\Delta}, T_{P}\right)$ is a triangulation of $\left(\mathbb{R}^{q}, M, \Delta, P\right)$,

$P_{0}$ is the $(p-1)$-skeleton of $T_{P}$,

$N:=N\left(P, T_{M}^{\prime \prime}\right), N_{0}:=N\left(P_{0}, T_{M}^{\prime \prime}\right)$,

$W:=N\left(P, T^{\prime \prime}\right), W_{0}:=N\left(P_{0}, T^{\prime \prime}\right)$,

$D:=M-$ int $N$,

$\sigma_{i}(i=1,2, \ldots)$ are the $p$-simplices of $T_{P}$,

$\left(B_{i}, A_{i}\right)$ is the canonical $p$-handle of $(W, N)$ (i.e. the closure of the component of $\left.\left(W-W_{0}, N-N_{0}\right)\right)$ intersecting $\sigma_{i}$,

$C_{i}:=A_{i} \cap \sigma_{i}$ is the core of $\left(B_{i}, A_{i}\right)$, and

$\pi_{p}:=\pi_{p} E\left(D^{m-p}, D^{m}\right)$.

In addition let $V:=W \times D^{1} \subset \mathbb{R}^{q} \times \mathbb{R}=\mathbf{R}^{q+1}$. Choose a collar on $\partial V$ in $\mathbf{R}^{q+1}-$ int $V:$ let $h: I \times \partial V \rightarrow \mathbf{R}^{q+1}-$ int $V$ be an embedding such that

$h(0, x)=x$ for each $x \in \partial V$,

$h(I \times \partial W) \subset \mathbf{R}^{q}$ (here $\left.W \equiv W \times 0\right)$,

$h(I \times \partial N)=M_{0}-$ int $N$,

$h(t,(w, 1))=(w, t+1)$ for each $w \in W$ and $t \in I$,

$h(I \times \partial V) \subset \mathbf{R}^{q} \times(-\infty, 2]$.

Denote by $\mathscr{E}_{0}^{q}$ the set of all $e \in \mathscr{E}^{q}$ that satisfy the following conditions:

$e \mid P \cup N_{0}=\mathrm{id}$,

$e\left(A_{i}\right)$ is properly embedded in $B_{i}$, for each $i$,

$e(h(t, x))=h(t, e(x))$ for each $t \in I$ and $x \in \partial N$,

$e($ int $\Delta) \subset \mathbf{R}^{q}-W-h(I \times \partial W)$.

Let $\mathscr{E}_{0}^{q+1}$ be the set of all $e \in \mathscr{E}^{q+1}$ such that $e \mid N=\mathrm{id}, e($ int $D) \subset \mathbf{R}^{q+1}-V$, and $e(D) \cap\left(P_{0} \times \mathbf{R}_{+}\right)=\varnothing$. 
3.4. Lemma. The composites $\mathscr{E}_{0}^{q} \hookrightarrow \mathscr{E}^{q} \rightarrow \mathscr{I}^{q}$ and $\mathscr{E}_{0}^{q+1} \hookrightarrow \mathscr{E}^{q+1} \rightarrow \mathscr{I}^{q+1}$ are surjective.

Proof. The proof of the second assertion is similar to the proof of Lemma 2.7: given any $e \in \mathscr{E}^{q+1}$ we use the fact that $N$ unknots in $\mathbf{R}^{q+1}$ to deform $e$ to an embedding which is identity on $N$, and then we use uniqueness of relative regular neighborhoods and general position to get the image of int $D$ into $\mathbb{R}^{q+1}-V-\left(P_{0} \times \mathbb{R}_{+}\right)$. To prove the first assertion of 3.4 we can start with an embedding $e$ in the set $\mathscr{E}_{0}\left(M, \mathbb{R}^{q}\right)$ of Lemma 2.7. We use uniqueness of relative regular neighborhoods to get the image of $h(I \times \partial N)$ into $h(I \times \partial W)$ and the image of int $\Delta$ into $\mathbf{R}^{q}-W-h(I \times \partial W)$. Then we apply Hudson's Theorem 1.1 of [5] to "straighten" the image of $h(I \times \partial N)$ in $h(I \times \partial W)$.

Proof of Theorem 3.1. Let us recall the definition of the bijection $\delta$. Orient $M$. Take any isotopy class $\varepsilon \in \mathscr{J}^{q+1}$ and pick an $e \in \varepsilon \cap \mathscr{E}_{0}^{q+1}$. Thinking of $e(D) \cup(-D)$ as an $m$-cycle in $\mathbb{R}^{q+1}-P$ let $\vartheta(e) \in H^{p}\left(P ; \mathbb{Z}_{d}\right)$ be the Alexander dual of $[e(D) \cup(-D)] \in H_{m}\left(\mathbb{R}^{q+1}-P ; \mathbb{Z}_{d}\right)$. It was proved in [14, 7.1] that $\vartheta(e)$ depends only on $\varepsilon$ and that by setting $\vartheta_{*}(\varepsilon):=\vartheta(e)$ we get a bijection $\vartheta_{*}: \mathscr{J}^{q+1} \nrightarrow H^{p}\left(P ; \mathbb{Z}_{d}\right)$. The function $\delta$ is then the composite of $\vartheta_{*}$ and the canonical isomorphism $H^{p}\left(P ; \mathbb{Z}_{d}\right) \approx H_{k+1}\left(M ; \mathbb{Z}_{d}\right)$ (see (2.3)).

The Alexander dual of an $m$-cycle $z$ in $R^{q+1}-P$ can be calculated as follows (cf. the proof of Lemma 2.11). Let $c P$ be the rectilinear join of $P$ with a point $c$ on the ray $0^{q} \times \mathbf{R}_{+}$. If the support of $z$ misses $c P_{0}$, then the Alexander dual of $[z] \in H_{m}\left(\mathbb{R}^{q+1}-P ; \mathbb{Z}_{d}\right)$ is, up to sign, the cohomology class of the simplicial $p$-cocycle on $T_{P}$ which assigns to each oriented $p$-simplex $\sigma_{i} \in T_{P}$ the intersection number $\operatorname{sc}\left(c \sigma_{i}, z ; \mathbf{R}^{q+1}\right) \bmod d$. Here it will be convenient to push the point $c$ to "infinity", i.e. we shall replace the cone $c P$ with $P \times \mathbf{R}_{+}$. Therefore $\vartheta_{*}(\varepsilon)=\vartheta(e)$ is up to sign the cohomology class of the simplicial p-cocycle

$$
\sigma_{i} \mapsto \operatorname{sc}\left(\sigma_{i} \times \mathbb{R}_{+}, e(D) ; \mathbb{R}^{q+1}\right) \bmod d
$$

To prove Theorem 3.1 we must be able to evaluate $\vartheta_{*}$ on the isotopy class (in $\mathbf{R}^{q+1}$ ) of an arbitrary $e \in \mathscr{E}^{q}$ (actually it suffices to consider embeddings $\left.e \in \mathscr{E}_{0}^{q}\right)$. This cannot be done immediately since $\vartheta(e)$ is not defined for all $e$; we must first deform $e$ (by an isotopy in $\mathbf{R}^{q+1}$ ) to an embedding $e^{\prime}$ belonging to $\mathscr{E}_{0}^{q+1}$.

Take, therefore, an arbitrary $e \in \mathscr{E}_{0}^{q}$. An isotopy $G: I \times M \rightarrow I \times \mathbb{R}^{q+1}$ from $e$ to an $e^{\prime} \in \mathscr{E}_{0}^{q+1}$ is constructed as follows. Let $G_{t}(x):=e(x)$ for all $t \in I$ if $x$ is in one of $P, N_{0}$, or $\Delta$. For each $p$-handle $A_{i}$ of $N$ let $G \mid I \times A_{i}$ be an arbitrary proper isotopy of $A_{i}$ in $B_{i} \times D^{1}$ (keeping $C_{i}$ and $A \cap N_{0}$ fixed) such that $G_{0}\left|A_{i}=e\right| A_{i}$ and $G_{1} \mid A_{i}=\mathrm{id}$; that such an isotopy exists follows from 
Theorem 1.9. Finally, on the collar $h(I \times \partial N)$ define $G_{t}(0 \leq t \leq 1)$ by

$$
G_{t}(h(s, x)):= \begin{cases}h(s, e(x)) & \text { if } t \leq s, \\ h\left(s, G_{t-s}(x)\right) & \text { if } t \geq s .\end{cases}
$$

Note that the embedding $e^{\prime}:=G_{1}: M \rightarrow \mathbb{R}^{q+1}$ belongs to $\mathscr{E}_{0}^{q+1}$ and is given by

$$
e^{\prime}(x)= \begin{cases}x & \text { if } x \in N, \\ h(j \times \mathrm{id}) G(j \times \mathrm{id})(s, y) & \text { if } x=h(s, y) \in h(I \times \partial N), \\ e(x) & \text { if } x \in \Delta,\end{cases}
$$

where $j: I \rightarrow I$ is the reflection $j(s):=1-s$. It follows that, up to sign, $\vartheta\left(e^{\prime}\right)$ is the cohomology class of the simplicial $p$-cocycle (see (3.5)):

$$
\sigma_{i} \mapsto \operatorname{sc}\left(C_{i} \times[1,2], e^{\prime} h\left(I \times\left(D \cap A_{i}\right)\right) ; h(I \times \partial V)\right) \bmod d .
$$

Observe that

$$
\begin{gathered}
C_{i} \times[1,2]=h\left(I \times\left(C_{i} \times 1\right)\right)=h(j \times \mathrm{id})\left(I \times\left(C_{i} \times 1\right)\right) \\
e^{\prime} h\left(I \times\left(D \cap A_{i}\right)\right)=h(j \times \mathrm{id}) G(j \times \mathrm{id})\left(I \times\left(D \cap A_{i}\right)\right) \\
=h(j \times \mathrm{id}) G\left(I \times\left(D \cap A_{i}\right)\right) \\
h(I \times \partial V)=h(j \times \mathrm{id})(I \times \partial V) .
\end{gathered}
$$

It follows that a representative cocycle for $\vartheta\left(e^{\prime}\right)$ is given by

$$
\sigma_{i} \mapsto \pm \operatorname{sc}\left(I \times C_{i} \times 1, G\left(I \times\left(D \cap A_{i}\right)\right) ; I \times \partial V\right) \bmod d
$$

where the sign is independent of $i$.

Clearly, the embedding $e$ does not determine the isotopy $G$ uniquely. Nevertheless, choosing for each $e \in \mathscr{E}_{0}^{q}$ one such $G$ arbitrarily we get a function $\beta: \mathscr{E}_{0}^{q} \rightarrow \mathscr{E}_{0}^{q+1}\left(e \mapsto e^{\prime}=G_{1}\right)$. Formula (3.6) for a representative cocycle of $\vartheta \beta(e)$ and Proposition 1.17 imply that the following diagram commutes up to sign (the set $\mathscr{E}_{0}(N, W)$ was defined just before Lemma 2.7, the function [z] in Proposition 2.8, and $r$ is the restriction):

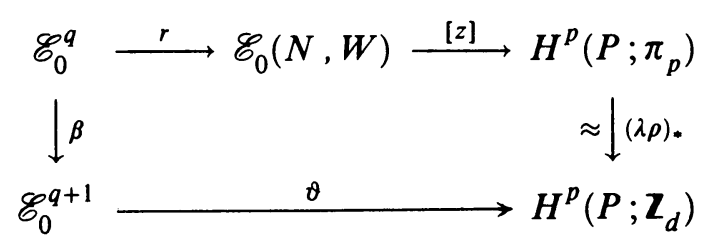

As each $e \in \mathscr{E}_{0}^{q}$ is isotopic to $\beta(e)$, Theorem 3.1 is now an easy consequence of Proposition 2.8, the definition of $\gamma$ at the end of the proof of Theorem 2.1, and Lemma 3.4.

A variant of the proof of Theorem 3.1 proves the next theorem. Here $M$ is assumed to have the same intrinsic properties as before, but we do not assume that $M$ lies in (or can be embedded into) $\mathbf{R}^{q}$. 
3.7 Theorem. Every embedding of $M$ in $\mathbf{R}^{q+1}$ is isotopic to an embedding $e: M \rightarrow \mathbf{R}^{q+1}$ such that $e\left(M_{0}\right) \subset \mathbf{R}^{q}$ and $e($ int $\Delta) \subset$ int $\mathbf{R}_{-}^{q+1}$. Two embeddings with the stated properties are isotopic in $\mathbf{R}^{q+1}$ if and only if their restrictions to $M_{0}$ are isotopic in $\mathbf{R}^{q}$.

Proof. Choose an embedding of $M_{0}$ in $\mathbf{R}^{q}[4,8.3$, p. 181] and extend it to an embedding $e_{0}: M \rightarrow \mathbf{R}^{q+1}$ such that $e_{0}$ (int $\left.\Delta\right) \subset$ int $\mathbf{R}_{-}^{q+1}$. From now on we shall identify $M$ with $e_{0}(M)$.

Assume the notation and the definitions given after the statement of Corollary 3.3. Let $\mathscr{E}_{1}^{q+1}$ be the set of all embeddings $M \rightarrow \mathbf{R}^{q+1}$ which send int $\Delta$ into int $R_{-}^{q+1}$ and embed $M_{0}$ into $R^{q}$ in the same way as the embeddings belonging to $\mathscr{E}_{0}^{q}$ do. Define $\beta_{1}: \mathscr{E}_{1}^{q+1} \rightarrow \mathscr{E}_{0}^{q+1}$ exactly as the function $\beta$ in the proof of Theorem 3.1 and let $r_{1}: \mathscr{E}_{1}^{q+1} \rightarrow \mathscr{E}_{0}(N, W)$ be the (obviously surjective) restriction map. Then $(\lambda \rho)_{*}[z] r_{1}= \pm \vartheta \beta_{1}$, which is verified in the same way as the relation $(\lambda \rho)_{*}[z] r= \pm \vartheta \beta$ at the end of the proof of Theorem 3.1. Hence, and by Proposition 2.8, the following diagram is commutative up to sign:

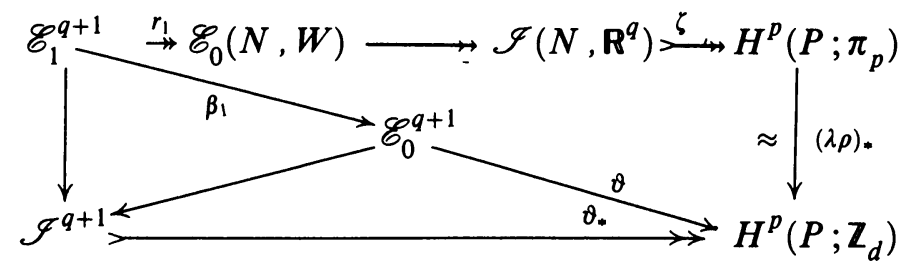

Theorem 3.7 follows.

Very similarly one can prove, referring to $[14,1.4]$, the following theorem:

3.8. Theorem. Let $Q$ be an $(m-k)$-connected $(q+1)$-manifold without boundary and $R$ be $a(q+1)$-disk in $Q$. Then every embedding of $M$ in $Q$ is isotopic to an embedding $e: M \rightarrow Q$ such that $e\left(M_{0}\right) \subset \partial R$ and $e$ (int $\left.\Delta\right) \subset Q-R$. $T$ wo embeddings with these properties are isotopic in $Q$ if and only if they are homotopic in $Q$ and their restrictions to $M_{0}$ are isotopic in $\partial R$.

\section{REFERENCES}

1. D. Burghelea, R. Lashof, and M. Rothenberg, Groups of automorphisms of manifolds, Lecture Notes in Math., vol. 473, Springer-Verlag, Berlin, 1975.

2. M. M. Cohen, A general theory of relative regular neighborhoods, Trans. Amer. Math. Soc. 136 (1969), 189-230.

3. A. Haefliger and V. Poenaru, La classification des immersions combinatoires, Inst. Hautes Études Sci. Publ. Math. 23 (1964), 75-91.

4. J. F. P. Hudson, Piecewise linear topology, Benjamin, New York, 1969.

5. __ Concordance, isotopy, and diffeotopy, Ann. of Math. (2) 91 (1970), 425-448.

6. L. S. Husch, Suspensions of PL-embeddings, Bull. London Math. Soc. 2 (1970), 191-195.

7. R. Lashof, Embedding spaces, Illinois J. Math. 20 (1976), 144-154.

8. C. Morlet, Les méthodes de la topologie différentielle dans l'étude des variétés semi-linéaires, Ann. Sci. École Norm. Sup. (4) 1 (1968), 313-394. 
9. R. D. Rigdon, Immersions and embeddings of manifolds in Euclidean space, Thesis, Univ. of California, Berkeley, 1970.

10. C. P. Rourke and B. J. Sanderson, Block bundles. II, Ann. of Math. (2) 87 (1968), 256-278.

11. __ Block bundles. III, Ann. of Math. (2) 87 (1968), 431-483.

12. G. P. Scott, A note on piecewise-linear immersions, Quart. J. Math. Oxford (2) 21 (1970), 257-263.

13. R. S. Tindell, Knotting tori in hyperplanes (J. G. Hocking, ed.), Conf. on Topology of Manifolds, Prindle, Weber \& Schmidt, 1968, pp. 147-153.

14. J. Vrabec, Knotting a k-connected closed PL m-manifold in $E^{2 m-k}$, Trans. Amer. Math. Soc. 233 (1977), 137-165.

15. C. T. C. Wall, Geometrical connectivity. I, J. London Math. Soc. (2) 3 (1971), 597-604.

16. T. Yasui, On the map defined by regarding embeddings as immersions, Hiroshima Math. J. 13 (1983), 457-467.

Department of Mathematics, University of Luubljana, P. P. 64, 61111 Ljubluana, YuGOSLAVIA 